Homology, Homotopy and Applications, vol.10(3), 2008, pp.101-135

\title{
THE RING SPECTRUM $P(n)$ FOR THE PRIME 2
}

\author{
J. MICHAEL BOARDMAN
}

\author{
(communicated by Haynes R. Miller)
}

Abstract

This paper is a companion to the Boardman-Wilson paper on the ring spectrum $P(n)$. When the prime is 2 , this spectrum is not commutative, which introduces several complications. Here, we supply the necessary details of the relevant Hopf algebroids and Hopf ring for this case.

\section{Introduction}

It is well known that the ring spectrum $P(n)$ for the prime 2 is not commutative. The purpose of this paper is to deal with the resulting complications by supplying the details that were deferred from [BW07]. While some results carry over from the odd prime case (though sometimes non-obviously), other results require extensive modification. We adopt much notation from [ibid.].

We recall that the Brown-Peterson spectrum $B P$ for the prime 2 has the coefficient ring $B P_{*}=\mathbb{Z}_{(2)}\left[v_{1}, v_{2}, v_{3}, \ldots\right]$, a polynomial ring over $\mathbb{Z}_{(2)}$ (the integers localized at 2 ) on generators $v_{i}$ in degree $2\left(2^{i}-1\right)$. The ring spectrum $P(n)$ is constructed [BW07, $\S 2]$ from $B P$ to have the coefficient ring

$$
P(n)_{*}=B P_{*} / I_{n}=\mathbb{F}_{2}\left[v_{n}, v_{n+1}, v_{n+2}, \ldots\right],
$$

where $I_{n}$ denotes the ideal $\left(2, v_{1}, v_{2}, \ldots, v_{n-1}\right)$ and $0<n<\infty$. (We are not concerned with the commutative ring spectra $P(0)=B P$ and $P(\infty)=H\left(\mathbb{F}_{2}\right)$.) We work in characteristic 2 . Tensor products are taken over $P(n)_{*}$. Because it occurs frequently, we find it useful to write $N=2^{n}-1$.

As most of the work is in homology, we use homology degrees throughout (unlike [BJW95]): elements of the coefficient group $P(n)_{i}$ and homology classes in $P(n)_{i}(X)$ have degree $i$, which forces cohomology classes in $P(n)^{i}(X)$ to have degree $-i$.

In $\S 2$, we verify, following Nassau [Na02], that there are exactly two good multiplications on $P(n)$ and discuss their properties. In $\S 3$, we examine what happens to the Künneth formula and universal coefficient theorem in some generality. In $\S 4$, we specialize to $P(n)$; most standard results survive unchanged, but not all.

In $\S 5$, we recall from [BW07] the Hopf algebroid $\Gamma$ that encodes the stable operations in $P(n)$-cohomology, the bigraded Hopf algebroid $A_{*}^{*}$ that encodes the additive operations, and the Hopf ring that encodes the unstable operations. This Hopf ring

Received February 22, 2008, revised March 24, 2008; published on December 5, 2008.

2000 Mathematics Subject Classification: 55P43, 55N22.

Key words and phrases: cohomology, Hopf ring.

This article is available at http://intlpress.com/HHA/v10/n3/a6

Copyright (c) 2008, International Press. Permission to copy for private use granted. 
is not commutative, which makes it necessary in $\S 6$ to be more precise about its structure. In $\S 7$, we prove the theorems stated in $\S 6$.

In $\S 8$, we develop a nonadditive idempotent cohomology operation to complete a proof in [BW07]. To establish its existence, in $\S 9$ we develop a concrete description of the Hopf ring.

The author thanks the referee for suggestions on improving the presentation.

\section{The spectrum $P(n)$}

Here, we work in the graded stable homotopy category Stab $b_{*}$. The construction [BW07, §2] of $P(n)$ makes it canonically a $B P$-module, equipped with an action of the exterior algebra $E\left(Q_{0}, Q_{1}, \ldots, Q_{n-1}\right)$ over $P(n)_{*}$, where $Q_{i}: P(n) \rightarrow P(n)$ is a map of $B P$-modules of degree $-\left(2^{i+1}-1\right)$. The most important of these, $Q_{n-1}$, has degree $-N=-\left(2^{n}-1\right)$ and is easily described [Na02] in terms of the exact triangle

$$
P(n-1) \stackrel{v_{n-1}}{\longrightarrow} P(n-1) \stackrel{\rho}{\longrightarrow} P(n) \stackrel{\delta}{\longrightarrow} P(n-1)
$$

as the composite $Q_{n-1}=\rho \circ \delta$. Given a multi-index $I=\left(i_{0}, i_{1}, \ldots i_{n-1}\right)$, where each $i_{r}$ is 0 or 1 , we write $Q^{I}$ for the composite $Q_{0}^{i_{0}} \circ Q_{1}^{i_{1}} \circ \cdots \circ Q_{n-1}^{i_{n-1}}$. If we put $|I|=$ $-\operatorname{deg} Q^{I}$, we see that

$$
|I|=\sum_{r=0}^{n-1} i_{r}\left(2^{r+1}-1\right) \leqslant 2^{n+1}-2-n=2 N-n<2 N=\operatorname{deg} v_{n} .
$$

We have the canonical unit $\eta: S \rightarrow B P \rightarrow P(n)$, where $S$ denotes the sphere spectrum. As in [BW07], we choose a fixed multiplication $\phi: P(n) \wedge P(n) \rightarrow P(n)$ that satisfies all the axioms 2.1 of [ibid.] except commutativity; this includes the derivation formula

$$
Q_{i} \circ \phi=\phi \circ\left(Q_{i} \wedge \mathrm{id}+\mathrm{id} \wedge Q_{i}\right): P(n) \wedge P(n) \longrightarrow P(n)
$$

Since $\phi$ is associative, the iterated multiplication $\phi_{k}: P(n) \wedge P(n) \wedge \cdots \wedge P(n) \rightarrow$ $P(n)$ is well defined for $k \geqslant 3$.

We need the opposite ring spectrum $\overline{P(n)}$, which is the same spectrum $P(n)$ but equipped with the opposite multiplication

$$
\bar{\phi}: P(n) \wedge P(n) \stackrel{T}{\longrightarrow} P(n) \wedge P(n) \stackrel{\phi}{\longrightarrow} P(n),
$$

where $T$ denotes the switch map. Mironov [Mi78, Thm. 4.7] computed it explicitly as

$$
\bar{\phi}=\phi \circ T=\phi+v_{n} \phi \circ\left(Q_{n-1} \wedge Q_{n-1}\right): P(n) \wedge P(n) \longrightarrow P(n) .
$$

Consequently, whenever two factors $P(n)$ are interchanged, this equation introduces an extra term. From now on, we write $Q=Q_{n-1}$, as the other $Q_{i}$ 's are of lesser interest. It follows immediately from equation (2) that $Q_{i}$ is also a derivation for $\bar{\phi}$,

$$
Q_{i} \circ \bar{\phi}=\bar{\phi} \circ\left(Q_{i} \wedge \mathrm{id}+\mathrm{id} \wedge Q_{i}\right): P(n) \wedge P(n) \longrightarrow P(n) .
$$


We next verify that there are exactly two good multiplications on $P(n)$, of which neither appears to be preferred. For $n \geqslant 3$, there are many uninteresting nonassociative multiplications on $P(n)$, such as

$$
\phi+v_{3} \phi \circ\left(Q^{1,1,0} \wedge Q^{0,1,1}\right): P(3) \wedge P(3) \longrightarrow P(3) .
$$

Proposition 2.1. There are exactly two associative BP-bilinear multiplications on $P(n)$ that have $\eta$ as unit, namely $\phi$ and $\bar{\phi}$.

Proof. By [BW07, Lemma 2.2(ii)], any BP-bilinear multiplication on $P(n)$ that has $\eta$ as unit has the form

$$
\phi^{\prime}=\phi+\sum_{I, J} c_{I, J} \phi \circ\left(Q^{I} \wedge Q^{J}\right): P(n) \wedge P(n) \longrightarrow P(n)
$$

with $c_{I, J} \in P(n)_{*}$, where all multi-indices $I$ etc. are understood to be nonzero. (The term $\phi$ must be present to make $\eta$ a unit, as $Q_{i} \circ \eta=0$ trivially. By (1), there are no terms with $c_{I, 0}$ or $c_{0, J}$; further, each $c_{I, J}$ must be $v_{n}$ or zero.)

With the help of (2), we expand the iterated product of $\phi^{\prime}$ in the standard form $[$ BW07, (2.4)] as

$$
\begin{aligned}
& \phi^{\prime} \circ\left(\phi^{\prime} \wedge \mathrm{id}\right)= \phi_{3}+\sum_{I, J} c_{I, J} \phi_{3} \circ\left(Q^{I} \wedge Q^{J} \wedge \mathrm{id}+Q^{I} \wedge \mathrm{id} \wedge Q^{J}\right. \\
&\left.+\mathrm{id} \wedge Q^{I} \wedge Q^{J}\right) \\
&+\sum_{I, J, K} c_{I+J, K} \phi_{3} \circ\left(Q^{I} \wedge Q^{J} \wedge Q^{K}\right) \quad(I \text { and } J \text { disjoint }) \\
&+ \text { terms with } v_{n}^{2},
\end{aligned}
$$

where we call $I$ and $J$ disjoint if $Q^{I}$ and $Q^{J}$ have no common factor. Similarly, $\phi^{\prime} \circ\left(\mathrm{id} \wedge \phi^{\prime}\right)$ has the same form, except that the second sum is replaced by

$$
\sum_{I, J, K} c_{I, J+K} \phi_{3} \circ\left(Q^{I} \wedge Q^{J} \wedge Q^{K}\right) \quad(J \text { and } K \text { disjoint }) .
$$

For $\phi^{\prime}$ to be associative, we must have $c_{I+J, K}=c_{I, J+K}$ for all $I, J, K$.

Suppose $c_{L, M}=v_{n}$. Then $L$ and $M$ cannot be disjoint, because if they were, (1) would imply $|L|+|M|=|L+M|<\operatorname{deg} v_{n}$. Suppose $Q^{L}$ and $Q^{M}$ have the common factor $Q_{k}$. We claim that $Q^{L}=Q_{k}$; otherwise, $Q^{L}=Q_{k} \circ Q^{P}$ with $P \neq 0$, and the term $v_{n} \phi_{3} \circ\left(Q^{P} \wedge Q_{k} \wedge Q^{M}\right)$ would appear in (5) but not in (6). Similarly, $Q^{M}=Q_{k}$. Finally, to make $|L|+|M|=\operatorname{deg} v_{n}$, we need $k=n-1$, which forces $\phi^{\prime}=\bar{\phi}$.

\section{Complex conjugation}

There is an alternate explanation for why $P(n)$ should behave differently when $p=2$.

Given any complex-oriented ring spectrum $E$ with a natural first Chern class $x(\xi) \in$ $E^{2}(-)$ for complex line bundles, one can define the complex conjugate Chern class $\bar{x}(\xi)=-x(\bar{\xi})$, where $\bar{\xi}$ denotes the complex conjugate line bundle (with a sign to make $\bar{x}$ a strict Chern class, if we are not in characteristic 2). Since $\xi \otimes \bar{\xi}$ is a trivial 
line bundle, we have the formula

$$
\bar{x}(\xi)=-[-1](x(\xi)),
$$

where the series $[-1](x)$ is defined in terms of the formal group law $F(x, y)$ of $x(-)$ by $F(x,[-1](x))=0$.

For $B P$, taking formal logarithms, we have $\log x+\log ([-1](x))=0$. If $p$ is odd, we have simply $[-1](x)=-x$, because all the exponents in the series $\log x$ are odd; then $\bar{x}(\xi)=x(\xi)$, leaving nothing to discuss. But for $p=2$, the series $[-1](x)$ is highly nontrivial. Now $P(n)$ inherits its complex orientation and Chern classes from $B P$, and $[-1](x)$ remains nontrivial for $P(n)$.

Nassau proved [Na02, Thm. 3] that the two good multiplications on $P(n)$ are abstractly isomorphic.

Theorem 2.2 (Nassau). There is a canonical antiautomorphism $\Xi$ of the ring spectrum $P(n)$, i.e. isomorphism $\Xi: P(n) \cong \overline{P(n)}$ of ring spectra, which is characterized by $\Xi x(\xi)=\bar{x}(\xi)$ in cohomology for any complex line bundle $\xi$.

In words, as a complex-oriented ring spectrum, $\overline{P(n)}$ is isomorphic to $P(n)$ equipped with the multiplication $\phi$ and the conjugate complex orientation.

More generally, we determine all automorphisms and antiautomorphisms. We recall from [BW07, Thm. 6.4(ii)] that when $p=2$, the Hopf algebroid for $P(n)$ is the polynomial ring

$$
\Gamma=\overline{P(n)}_{*}(P(n))=P(n)_{*}\left[a_{(0)}, a_{(1)}, \ldots, a_{(n-1)}, b_{(n+1)}, b_{(n+2)}, \ldots\right],
$$

where the missing elements $b_{(j)}$ are given by

$$
a_{(i)}^{2}=b_{(i+1)} \quad(\text { for } 0 \leqslant i \leqslant n-1) .
$$

As an abelian group, $\overline{P(n)}_{*}(P(n))$ is identical to $P(n)_{*}(P(n))$ (and even has the same $P(n)_{*}$-module structure, as we see in $\left.\S 4\right)$. The novelty here is that the multiplication is given by $c d=\phi_{*}(c \overline{\times} d)$, where $c \overline{\times} d$ denotes the cross product in $\overline{P(n)}$-homology.

As in [Bo95, (11.1)], we identify a stable operation $r: P(n) \rightarrow P(n)$ with its associated $P(n)_{*}$-linear functional $\langle r,-\rangle: \Gamma \rightarrow P(n)_{*}$. (We check in Proposition 4.5 that it still $i s$ linear.) The homomorphism $r_{*}: \Gamma \rightarrow \Gamma$ induced by $r$ on $\overline{P(n)}$-homology is also of interest. equation (11.33) of [ibid.] uses the coalgebra structure $\left(\psi_{S}, \epsilon_{S}\right)$ on $\Gamma$ to express $r_{*}$ in terms of $\langle r,-\rangle$ as

$$
r_{*}: \Gamma \stackrel{\psi_{S}}{\longrightarrow} \Gamma \otimes \Gamma \stackrel{\operatorname{id} \otimes\langle r,-\rangle}{\longrightarrow} \Gamma \otimes P(n)_{*} \cong \Gamma,
$$

where we use the right action of $P(n)_{*}$ on $\Gamma$. Conversely, we recover $\langle r,-\rangle$ from $r_{*}$ by equation (11.30) of [ibid.] as

$$
\langle r,-\rangle: \Gamma \stackrel{r_{*}}{\longrightarrow} \Gamma \stackrel{\epsilon_{S}}{\longrightarrow} P(n)_{*} .
$$

Theorem 2.3. An automorphism or antiautomorphism $r$ of the ring spectrum $P(n)$ is uniquely determined by the values $\left\langle r, b_{(j)}\right\rangle \in P(n)_{2\left(2^{j}-1\right)}$ for $j>n$ and the value $\left\langle r, b_{(n)}\right\rangle=0$ (for an automorphism) or $\left\langle r, b_{(n)}\right\rangle=v_{n}$ (for an antiautomorphism). The values may be chosen arbitrarily. 
Proof of Theorem 2.2. Since $\bar{x}$ is a 2-typical Chern class on $B P$, there is a unique map of ring spectra $\Xi: B P \rightarrow B P$ that induces $\Xi x=\bar{x}$. For $B P$, we have $[-1](x) \equiv x+$ $v_{n} x^{2^{n}}+\ldots \bmod I_{n}$; this follows from the standard fact [RW77, Thm. 3.11(b)] that $[2](x) \equiv v_{n} x^{2^{n}}+\ldots \bmod I_{n}$ and the formal group law identity $F([2](x),[-1](x))=$ $[1](x)=x$. From $\left[\right.$ Bo95, (13.3)] we have $\Xi x=\sum_{j}\left\langle\Xi, b_{j}\right\rangle x^{j}$. We compare this with $\bar{x}=[-1](x)$ to deduce $\left\langle\Xi, b_{(n)}\right\rangle=v_{n}$; then Theorem 2.3 shows that $\Xi: B P \rightarrow B P$ descends to an antiautomorphism of $P(n)$.

Theorem 2.3 will follow immediately from Lemma 2.4, below. For this, we need information on the induced homomorphism $r_{*}: P(n)_{*} \rightarrow P(n)_{*}$ of homotopy groups. The discussion in [BW07, §6.6] shows that for each $k \geqslant n$, the stable main relation $\left(\mathcal{R}_{k}\right)$ reduces to $w_{k} \equiv v_{k} 1 \bmod I_{k} \Gamma$, where $w_{k}=\eta_{R} v_{k}$ and $I_{k}$ denotes the ideal $\left(v_{n}, v_{n+1}, \ldots, v_{k-1}\right)$ in $P(n)_{*}$. Then by [Bo95, Prop. 11.22(c)], on homotopy groups

$$
r_{*} v_{k}=\left\langle r, w_{k}\right\rangle \equiv\left\langle r, v_{k} 1\right\rangle=v_{k}\langle r, 1\rangle \quad \bmod I_{k} .
$$

Lemma 2.4. Suppose that $r: P(n) \rightarrow P(n)$ is a map of spectra (or operation) of degree zero whose linear functional $\langle r,-\rangle: \Gamma \rightarrow P(n)_{*}$ satisfies:

(i) $\langle r, 1\rangle=1$;

(ii) $\left\langle r, b^{J+K}\right\rangle=\left\langle r, b^{J}\right\rangle\left\langle r, b^{K}\right\rangle$ for all multi-indices $J$ and $K$;

(iii) $\left\langle r, a^{I} b^{J}\right\rangle=0$ whenever $I$ is nonzero.

(a) If $\left\langle r, b_{(n)}\right\rangle=0, r$ is an automorphism of the ring spectrum $P(n)$, and every automorphism has this form;

(b) If $\left\langle r, b_{(n)}\right\rangle=v_{n}, r$ is an antiautomorphism of the ring spectrum $P(n)$, and every antiautomorphism has this form.

Proof. It is clear from diagrams (8) and (9) that $r$ is multiplicative if and only if $\langle r,-\rangle$ is. Because $a_{(i)}$ has odd degree, $\left\langle r, a_{(i)}\right\rangle=0$ for all $i$. For dimensional reasons, $\langle r,-\rangle$ automatically preserves the relation (7) for $i \leqslant n-2$, leaving only the case $i=n-1$ to check. If $\left\langle r, b_{(n)}\right\rangle=0,\langle r,-\rangle$ is multiplicative. Then (10) is enough to guarantee that $r$ is invertible, and we have (a).

For $r$ to be an antiautomorphism, we require the diagram

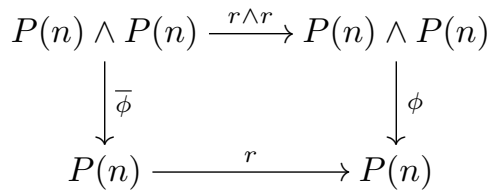

to commute. We apply $\overline{P(n)}$-homology to obtain the commutative diagram

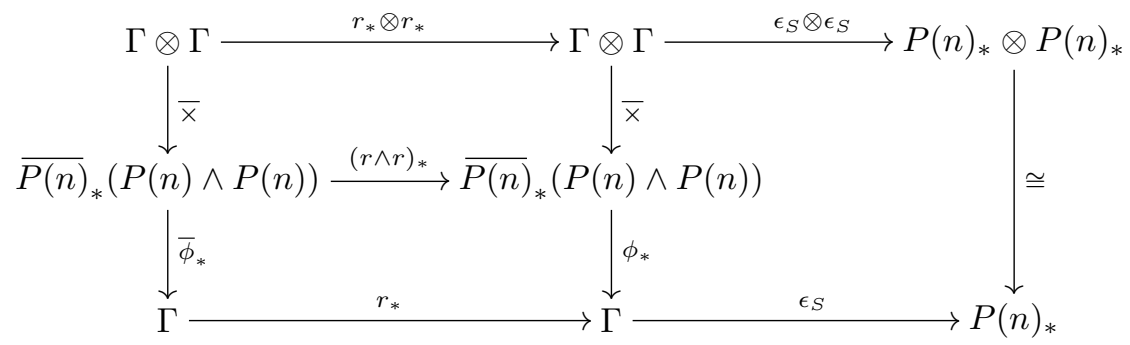


in which we recognize $\epsilon_{S} \circ r_{*}$ as $\langle r,-\rangle$ by (9). We evaluate on $c \otimes d \in \Gamma \otimes \Gamma$. The left side of the diagram is not the multiplication on $\Gamma$; instead, it sends $c \otimes d$ to $\bar{\phi}_{*}(c \overline{\times}$ $d)=c d+v_{n}\left(Q_{*} c\right)\left(Q_{*} d\right)$, by (3). We deduce that $\langle r,-\rangle$ must satisfy the identity

$$
\langle r, c d\rangle+v_{n}\left\langle r,\left(Q_{*} c\right)\left(Q_{*} d\right)\right\rangle=\langle r, c\rangle\langle r, d\rangle
$$

for all $c$ and $d$. We take $c=d=a_{(n-1)}$; since $Q_{*} a_{(n-1)}=1$ (see Proposition 5.1), we must have $\left\langle r, b_{(n)}\right\rangle=v_{n}$. Then (10) shows that $r$ is invertible, to complete (b).

\section{Noncommutative ring spectra}

In the following section, we consider what effects the noncommutativity of $P(n)$ for $p=2$ has on standard results. As preparation, in this section we find it useful for clarity to be vastly more general. We assume that $E$ is a ring spectrum with characteristic 2, associative multiplication $\phi: E \wedge E \rightarrow E$, and two-sided unit $\eta: S \rightarrow$ $E$, without any commutation rules. (The restriction to characteristic 2 is merely for convenience, as it covers our current examples. It can be removed by inserting appropriate signs, of which a few are less than obvious.) The biggest change is that $E$-(co)homology must now be treated as an $E_{*}$-bimodule.

We continue to work in the graded stable homotopy category $S t a b_{*}$, and understand all (co)homology in the reduced sense. Tensor products are now taken over $E_{*}$. We suppress the associativity equivalences for the smash product $\wedge$, but not those for commutativity $X \wedge Y \simeq Y \wedge X$ or unit $S \wedge X \simeq X \simeq X \wedge S$ equivalences. However, we do identify

$$
E_{i}=\pi_{i}^{S}(E)=E^{-i}(S)=\operatorname{Stab}_{i}(S, E) \cong \operatorname{Stab}_{i}(S, E \wedge S)=E_{i}(S)
$$

We economize by recycling the proofs in [Bo95], as most of the necessary commutative diagrams are unchanged. (Many of them can also be found in Adams [Ad69].) All we have to do is determine which actions are actually used in the old proofs, in the absence of commutativity.

\section{Labeled (co)homology}

Because $E$ is not commutative, we must keep track of how the various copies of $E$ are shuffled. To accomplish this, we adopt the Mattaponi ${ }^{1}$ naming convention, by decorating each copy of $E$ with a character string to indicate its provenance.

In detail, a given cohomology class $x \in E^{*}(X)$ becomes a map $x: X \rightarrow E_{x}$, a homology class $a \in E_{*}(X)$ becomes a map $a: S \rightarrow E_{a} \wedge X$, and an element $v \in E_{*}$ becomes a map $v: S \rightarrow E_{v}$. Further copies of $E$ are introduced (recursively) by multiplications $\phi: E_{A} \wedge E_{B} \rightarrow E_{A B}$ or the opposite multiplications $\bar{\phi}: E_{A} \wedge E_{B} \rightarrow E_{B A}$, where $A$ and $B$ may be any character strings. For example, $\phi_{3}: E_{a} \wedge E_{b} \wedge E_{c} \rightarrow E_{a b c}$ is well defined, and generally $\phi_{k}$. (One could be even more general and replace selected copies of $E$ by a left or right $E$-module, with no extra work.)

\footnotetext{
${ }^{1}$ In Virginia [US69], the Po and Ni Rivers join to form the Poni River, which in turn merges with the Matta River to form the Mattaponi River (pronounced Mattapon-EYE); the south-to-north order is respected throughout.
} 


\section{Cross products}

Given cohomology classes $x \in E^{*}(X)$ and $y \in E^{*}(Y)$, their cross product $x \times y \in$ $E^{*}(X \wedge Y)$ is

$$
x \times y: X \wedge Y \stackrel{x \wedge y}{\longrightarrow} E_{x} \wedge E_{y} \stackrel{\phi}{\longrightarrow} E_{x y}
$$

It is clearly associative and natural in $X$ and $Y$.

Similarly, given homology classes $a \in E_{*}(X)$ and $b \in E_{*}(Y)$, their cross product $a \times b \in E_{*}(X \wedge Y)$ is

$$
\begin{aligned}
a \times b: S & \simeq S \wedge S \stackrel{a \wedge b}{\longrightarrow} E_{a} \wedge X \wedge E_{b} \wedge Y \\
& \simeq E_{a} \wedge E_{b} \wedge X \wedge Y \stackrel{\phi \wedge X \wedge Y}{\longrightarrow} E_{a b} \wedge X \wedge Y .
\end{aligned}
$$

It, too, is associative and natural.

\section{Bimodule structure}

We next make $E^{*}(X)$ and $E_{*}(X)$ into $E_{*}$-bimodules.

We define the left action of $E_{*}$ on $E^{*}(X)$ as usual: given $x \in E^{*}(X)$ and $v \in E_{*}$, the element $v x \in E^{*}(X)$ corresponds to $v \times x \in E^{*}(S \wedge X)$ under the isomorphism $E^{*}(X) \cong E^{*}(S \wedge X)$. We similarly define the right action of $E_{*}$ by making $x v \in$ $E^{*}(X)$ correspond to $x \times v \in E^{*}(X \wedge S)$. In particular, $1 x=x=x 1$, as $1 \in E_{*}$ is $\eta: S \rightarrow E$. By associativity of $E,(v x) v^{\prime}=v\left(x v^{\prime}\right)$, and the two actions make $E^{*}(X)$ an $E_{*}$-bimodule; further,

$$
\times: E^{*}(X) \otimes E^{*}(Y) \longrightarrow E^{*}(X \wedge Y)
$$

becomes a homomorphism of $E_{*}$-bimodules (in the usual sense). Moreover, it remains continuous in the profinite topology on cohomology (see [Bo95, Defn. 4.9]) defined by filtering $E^{*}(X)$ by the subbimodules

$$
F^{\alpha} E^{*}(X)=\operatorname{Ker}\left[i_{\alpha}^{*}: E^{*}(X) \longrightarrow E^{*}\left(X_{\alpha}\right)\right]
$$

where $X_{\alpha}$ runs over the finite subspectra of $X$, included by $i_{\alpha}: X_{\alpha} \subset X$.

Similarly, we make $E_{*}(X)$ an $E_{*}$-bimodule and

$$
\times: E_{*}(X) \otimes E_{*}(Y) \longrightarrow E_{*}(X \wedge Y)
$$

a homomorphism of $E_{*}$-bimodules (except that homology is always discrete).

\section{The Künneth Theorem}

The proof of Theorem 4.2 in [Bo95] extends without change to this general context in the following form.

Theorem 3.1. Suppose that $E_{*}(X)$ is free or flat as a right $E_{*}$-module or that $E_{*}(Y)$ is free or flat as a left $E_{*}$-module. Then diagram (12) is an isomorphism of $E_{*}$ bimodules. 


\section{Twisted cross products}

Unfortunately, cross products are not enough. As we already saw in [BW07] and $\S 2$, we need also the twisted cross product $a \overline{\times} b \in E_{*}(X \wedge Y)$, defined as

$$
\begin{aligned}
a \overline{\times} b: S & \simeq S \wedge S \stackrel{a \wedge b}{\longrightarrow} E_{a} \wedge X \wedge E_{b} \wedge Y \\
& \simeq E_{a} \wedge E_{b} \wedge X \wedge Y \stackrel{\bar{\phi} \wedge X \wedge Y}{\longrightarrow} E_{b a} \wedge X \wedge Y .
\end{aligned}
$$

It is no longer a homomorphism of $E_{*}$-bimodules; instead, given $v \in E_{*}$, we find that

$$
\begin{aligned}
\text { (i) }(v a) \overline{\times} b & =a \overline{\times}(b v): S \longrightarrow E_{b v a} \wedge X \wedge Y ; \\
\text { (ii) }(a v) \overline{\times} b & =(a \overline{\times} b) v: S \longrightarrow E_{b a v} \wedge X \wedge Y ; \\
\text { (iii) } a \overline{\times}(v b) & =v(a \overline{\times} b): S \longrightarrow E_{v b a} \wedge X \wedge Y .
\end{aligned}
$$

(There are also twisted cross products in cohomology that we do not need here.)

\section{Scalar products}

Given $x \in E^{*}(X)$ and $a \in E_{*}(X)$, Adams [Ad69, p. 72] in effect defined their scalar (or Kronecker) product $\langle x, a\rangle \in E_{*}$ as

$$
\langle x, a\rangle: S \stackrel{a}{\longrightarrow} E_{a} \wedge X \stackrel{\mathrm{id} \wedge x}{\longrightarrow} E_{a} \wedge E_{x} \stackrel{\bar{\phi}}{\longrightarrow} E_{x a}
$$

being careful to end up in $E_{x a}$ in order to preserve the lexical order of $x$ and $a$. In our context, this ensures that, given $v \in E_{*}$,

(i) $\langle v x, a\rangle=v\langle x, a\rangle: S \longrightarrow E_{v x a}$;

(ii) $\langle x v, a\rangle=\langle x, v a\rangle: S \longrightarrow E_{x v a}$;

(iii) $\langle x, a v\rangle=\langle x, a\rangle v: S \longrightarrow E_{x a v}$.

Equivalently,

$$
\langle-,-\rangle: E^{*}(X) \otimes E_{*}(X) \longrightarrow E_{*}
$$

is another homomorphism of $E_{*}$-bimodules. In particular, taking $X=S$, we have $\langle v, w\rangle=v w$ for $v, w \in E_{*}$.

There is one surprise, caused by the shuffling of factors $E$. Given also $y \in E^{*}(Y)$ and $b \in E_{*}(Y)$, it is essential for our purposes in [BW07, Lemma 6.1] to expand $\langle x \times$ $y, a \times b\rangle$. However, $\langle x \times y, a \times b\rangle: S \longrightarrow E_{x y a b}$ does not simplify in this generality, and is not the same as $\langle x, a\rangle\langle y, b\rangle: S \longrightarrow E_{x a y b}$. But if we mix the cross products, we can show that

$$
\langle x \times y, a \overline{\times} b\rangle=\langle x,\langle y, b\rangle a\rangle: S \longrightarrow E_{x y b a} .
$$

Both sides easily reduce to the same map

$$
\begin{aligned}
S \simeq S \wedge S \stackrel{a \wedge b}{\longrightarrow} E_{a} \wedge X \wedge E_{b} \wedge Y \stackrel{\mathrm{id} \wedge x \wedge \mathrm{id} \wedge y}{\longrightarrow} \\
E_{a} \wedge E_{x} \wedge E_{b} \wedge E_{y} \simeq E_{x} \wedge E_{y} \wedge E_{b} \wedge E_{a} \stackrel{\phi_{4}}{\longrightarrow} E_{x y b a} .
\end{aligned}
$$

There is also the twisted scalar product $\overline{\langle x, a\rangle} \in E_{*}$, defined as

$$
\overline{\langle x, a\rangle}: S \stackrel{a}{\longrightarrow} E_{a} \wedge X \stackrel{\mathrm{id} \wedge x}{\longrightarrow} E_{a} \wedge E_{x} \stackrel{\phi}{\longrightarrow} E_{a x}
$$


with $\phi$ instead of $\bar{\phi}$. Its linearity properties are:

$$
\begin{aligned}
\text { (i) } \overline{\langle v x, a\rangle} & =\overline{\langle x, a v\rangle}: S \longrightarrow E_{a v x} \text {; } \\
\text { (ii) } \overline{\langle x v, a\rangle} & =\overline{\langle x, a\rangle} v: S \longrightarrow E_{a x v} \text {; } \\
\text { (iii) } \overline{\langle x, v a\rangle} & =v \overline{\langle x, a\rangle}: S \longrightarrow E_{v a x} \text {; }
\end{aligned}
$$

and for $w \in E_{*}, \overline{\langle v, w\rangle}=w v$.

\section{Duality}

The advantage of the twisted scalar product is that given $x \in E^{*}(X)$, (iii) shows that $\overline{\langle x,-\rangle}: E_{*}(X) \rightarrow E_{*}$ is a homomorphism of left $E_{*}$-modules, whereas $\langle x,-\rangle$ is not. Denote by $\operatorname{Mod}_{*}$ the graded category of left $E_{*}$-modules; we define the duality homomorphism

$$
d=d_{X}: E^{*}(X) \longrightarrow D E_{*}(X)=\operatorname{Mod}_{*}\left(E_{*}(X), E_{*}\right)
$$

by $d x=d_{X} x=\overline{\langle x,-\rangle}: E_{*}(X) \rightarrow E_{*}$.

Equations (15) actually show more, that $d$ is a homomorphism of $E_{*}$-bimodules, provided we make the dual $D M=\operatorname{Mod}_{*}\left(M, E_{*}\right)$ of any $E_{*}$-bimodule $M$ into an $E_{*^{-}}$ bimodule by endowing it with the non-obvious actions defined on $f \in D M$ by

$$
(v f) m=f(m v), \quad(f v) m=(f m) v,
$$

for any $v \in E_{*}$ and $m \in M$.

As in [Bo95, Defn. 4.8], we define the dual-finite topology on the dual DM of any (discrete) left $E_{*}$-module $M$ by filtering $D M$ by the submodules

$$
F^{K} D M=\operatorname{Ker}[-\mid K: D M \longrightarrow D K],
$$

where $K$ runs over all finitely generated left submodules of $M$. This topology is useful mainly when $M$ is a free module, in which case $D M$ may be viewed as a cartesian product of copies of $E_{*}$ (with degree shifts), equipped with the product topology.

Note that the definition of $d_{X}$ is asymmetric, and that in this context, equation (17) makes $D M$ a right $E_{*}$-module, filtered by right submodules. Even if $M$ is a bimodule, $F^{K} D M$ will in general not be a subbimodule; in fact, we do not use the right $E_{*^{-}}$ action on $E_{*}(X)$ at all.

Theorem 3.2. Suppose $E_{*}(X)$ is a free left $E_{*}$-module. Then the homomorphism $d_{X}$ in (16) is a homeomorphism of filtered right $E_{*}$-modules. In particular, $E^{*}(X)$ is complete Hausdorff.

Proof. We apply the proof of Theorem 9.25 in [Bo95], specifically the commutative diagram

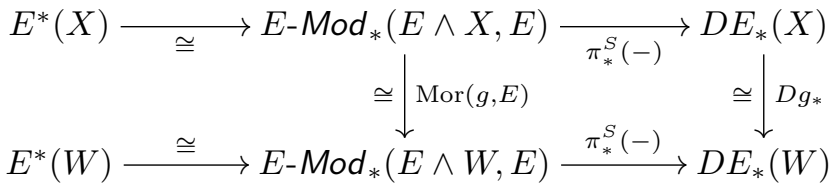

where $E-M_{o d}$ denotes the graded category of left $E$-module spectra, $W$ is a wedge of spheres, the rows are $d_{X}$ and $d_{W}$, and the vertical arrows are induced by an 
isomorphism $g: E \wedge W \rightarrow E \wedge X$ of left $E$-module spectra constructed from a suitable map $f: W \rightarrow E_{f} \wedge X$ of spectra. The proof given there still delivers a diagram of homeomorphisms.

All the horizontal arrows are isomorphisms of $E_{*}$-bimodules, using the $E_{*}$-actions on $D E_{*}(X)$ supplied by $(17)$ and on $E$ - $\operatorname{Mod}_{*}(E \wedge X, E)$ by transferring in the obvious way from $E^{*}(X)$. However, the resulting bijection $g^{*}: E^{*}(X) \cong E^{*}(W)$ is not an isomorphism of $E_{*}$-bimodules, and neither is $D g_{*}$. Given $x \in E^{*}(X)$, or map $x: X \rightarrow$ $E_{x}, g^{*} x$ is the composite

$$
g^{*} x: W \stackrel{f}{\longrightarrow} E_{f} \wedge X \stackrel{\mathrm{id} \wedge x}{\longrightarrow} E_{f} \wedge E_{x} \stackrel{\phi}{\longrightarrow} E_{f x} .
$$

Given also $v \in E_{*}$, or map $v: S \rightarrow E_{v}$, we compute that $g^{*}(x v)=\left(g^{*} x\right) v: W \rightarrow E_{f x v}$, showing that $g^{*}$ preserves the right $E_{*}$-action. In contrast, $v\left(g^{*} x\right)$ is the composite

$$
v\left(g^{*} x\right): W \simeq S \wedge W \stackrel{\mathrm{id} \wedge f}{\longrightarrow} S \wedge E_{f} \wedge X \stackrel{v \wedge \mathrm{id} \wedge x}{\longrightarrow} E_{v} \wedge E_{f} \wedge E_{x} \stackrel{\phi_{3}}{\longrightarrow} E_{v f x},
$$

while $g^{*}(v x)$ reduces to the same, but with $\phi_{3}$ replaced by

$$
E_{v} \wedge E_{f} \wedge E_{x} \stackrel{\bar{\phi} \wedge \mathrm{id}}{\longrightarrow} E_{f v} \wedge E_{x} \stackrel{\phi}{\longrightarrow} E_{f v x}
$$

which is different in general. Equations (17) show the same for $D g_{*}$, where the homomorphism $g_{*}: E_{*}(W) \rightarrow E_{*}(X)$ of left $E_{*}$-modules fails to preserve the right $E_{*}-$ action.

\section{Products in $P(n)$-(co)homology}

In this section, we specialize the results of the previous section by taking $E=P(n)$. From now on, we work mainly in the homotopy category Ho of unbased spaces and use absolute homology $P(n)_{*}(X)$ and cohomology $P(n)^{*}(X)$ (which may be viewed stably as the relative (co)homology of the pair $\left(X^{+}, o\right)$, where $X^{+}$denotes the disjoint union of $X$ and a (new) basepoint $o$ ).

As before, we identify the stable map $Q: P(n) \rightarrow P(n)$ with the stable operation $Q$ on $P(n)$-cohomology. Unstably, the space $\underline{P(n)}$ s represents the cohomology the-

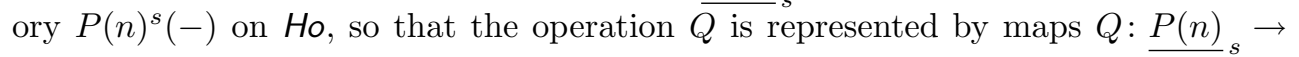
$\underline{P(n)}_{s+N^{*}}$.

The companion homology operation $Q$ is defined (stably) on $a \in P(n)_{*}(X)$ as

$$
Q a: S \stackrel{a}{\longrightarrow} P(n) \wedge X \stackrel{Q \wedge \mathrm{id}}{\longrightarrow} P(n) \wedge X .
$$

For the twisted cross product, equation (3) immediately yields

$$
T_{*}(a \times b)=b \overline{\times} a=b \times a+v_{n} Q b \times Q a \quad\left(\text { in } P(n)_{*}(Y \times X)\right)
$$

for any $a \in P(n)_{*}(X)$ and $b \in P(n)_{*}(Y)$, or equivalently,

$$
T_{*}(b \overline{\times} a)=a \times b=a \overline{\times} b+v_{n} Q a \overline{\times} Q b .
$$

For the cup product in $P(n)^{*}(X)$, we have the commutation rule

$$
y x=x y+v_{n}(Q x)(Q y) .
$$




\section{Modules}

As the ring $P(n)_{*}$ is commutative, there is no algebraic distinction between left $P(n)_{*}$-modules and right $P(n)_{*}$-modules. What we actually need is a stronger statement.

Proposition 4.1. The right action of $P(n)_{*}$ on $P(n)^{*}(X)$ and $P(n)_{*}(X)$ coincides with the left action: $x v=v x$ and $a v=v a$ for $v \in P(n)_{*}, x \in P(n)^{*}(X)$ and $a \in$ $P(n)_{*}(X)$. Moreover, the action of $P(n)_{*}$ is independent of the choice of the multiplication $\phi$ or $\bar{\phi}$ on $P(n)$.

Proof. If we take $Y$ to be a point in (18), we see that

$$
T_{*}(a \times v)=v \times a+v_{n} Q v \times Q a=v \times a,
$$

as $Q v=0$ trivially in $P(n)_{*}$ (point $)=P(n)_{*}$. Hence $a v=v a$. The analogous formula for cohomology (see [BW07, §2.2]) yields $x v=v x$.

By (18), changing $\phi$ to $\bar{\phi}$ adds the zero term $v_{n}(Q v)(Q a)$ to $v a$, and similarly for cohomology.

\section{Products}

Proposition 4.1 allows us to dispense with the right action of $P(n)_{*}$. The bimodule homomorphisms (11) and (12) simply reduce to the usual $P(n)_{*}$-bilinear homomorphisms in cohomology,

$$
\times: P(n)^{*}(X) \otimes P(n)^{*}(Y) \longrightarrow P(n)^{*}(X \times Y),
$$

and homology,

$$
\times: P(n)_{*}(X) \otimes P(n)_{*}(Y) \longrightarrow P(n)_{*}(X \times Y) .
$$

Thus the Künneth formula, Theorem 3.1, reverts to its traditional form, as stated in [Bo95, Thm. 4.2].

Theorem 4.2. If $P(n)_{*}(X)$ or $P(n)_{*}(Y)$ is a free or flat $P(n)_{*}$-module, the cross product induces an isomorphism

$$
\times: P(n)_{*}(X) \otimes P(n)_{*}(Y) \stackrel{\cong}{\longrightarrow} P(n)_{*}(X \times Y)
$$

of $P(n)_{*}$-modules, even for $p=2$.

Proposition 4.3. The (co)homology operation $Q$ behaves as expected on products:

(a) Given $a \in P(n)_{*}(X)$ and $b \in P(n)_{*}(Y)$, we have

$$
Q(a \times b)=Q a \times b+a \times Q b .
$$

We may replace $\times$ throughout by the twisted cross product $\overline{\times}$.

(b) Given $x \in P(n)^{*}(X)$ and $y \in P(n)^{*}(Y)$, we have

$$
Q(x \times y)=Q x \times y+x \times Q y .
$$

Again, we may replace $\times$ throughout by $\bar{x}$.

Proof. Both parts follow directly from (2) and (4).

The stable operation $Q$ is automatically additive. More is true. 
Corollary 4.4. The homology operation $Q: P(n)_{*}(Y) \rightarrow P(n)_{*}(Y)$ and cohomology operation $Q: P(n)^{*}(Y) \rightarrow P(n)^{*}(Y)$ are $P(n)_{*}$-linear homomorphisms.

Proof. We take $X$ as a point in Proposition 4.3 and $a=v \in P(n)_{*}$ (point) $=P(n)_{*}$. Since $Q v=0$ trivially, (21) reduces to $Q(v \times b)=v \times Q b$ and hence $Q(v b)=v Q b$.

The proof for cohomology is algebraically the same.

Scalar products also simplify in the same way.

Proposition 4.5. For $p=2$, given $x \in P(n)^{*}(X)$ and $a \in P(n)_{*}(X)$ :

(a) The scalar product $\langle x, a\rangle$ is $P(n)_{*}$-bilinear;

(b) The scalar product $\langle x, a\rangle$ is independent of the choice of multiplication $\phi$ or $\bar{\phi}$ on $P(n)$, and coincides with the twisted scalar product $\overline{\langle x, a\rangle}$ defined in $\S 3$;

(c) The operation $Q$ is self-adjoint, in the sense that $\langle Q x, a\rangle=\langle x, Q a\rangle$.

Proof. For (a), Proposition 4.1 allows us to treat (13) as being bilinear in the ordinary sense. Because $Q$ acts trivially on $P(n)_{*}$, (4) yields $0=Q\langle x, a\rangle=\langle Q x, a\rangle+$ $\langle x, Q a\rangle$, which gives (c). Then in (b), by (3), the two candidates for $\langle x, a\rangle$ differ by $v_{n}\langle Q x, Q a\rangle=v_{n}\langle x, Q Q a\rangle=0$.

We can now deduce the following result, which is Proposition 2.4 in [BW07].

Proposition 4.6. Given elements $x \in P(n)^{*}(X), y \in P(n)^{*}(Y), a \in P(n)_{*}(X)$ and $b \in P(n)_{*}(Y)$, we have

$$
\langle x \times y, a \times b\rangle=\langle x, a\rangle\langle y, b\rangle+v_{n}\langle x, Q a\rangle\langle Q y, b\rangle .
$$

If instead we mix the products, we find

$$
\langle x \times y, a \overline{\times} b\rangle=\langle x, a\rangle\langle y, b\rangle .
$$

Proof. Proposition 4.1 allows us to extricate $\langle y, b\rangle$ from its enclosing scalar product in (14) and rewrite that equation as (23). If we replace $a \overline{\times} b$ by $a \times b,(19)$ provides the extra term $v_{n}\langle x, Q a\rangle\langle y, Q b\rangle$. Then Proposition 4.5(c) allows us to replace $\langle y, Q b\rangle$ by $\langle Q y, b\rangle$ in order to obtain the more natural-looking (22).

\section{Duality}

In view of Propositions 4.5 and 4.1, Theorem 3.2 for $P(n)$ also reverts to its standard form, as stated in [Bo95, Thm. 4.14].

Theorem 4.7. Even for $p=2$, if $P(n)_{*}(X)$ is a free $P(n)_{*}$-module,

$$
d_{X}: P(n)^{*}(X) \cong D P(n)_{*}(X)=\operatorname{Mod}_{*}\left(P(n)_{*}(X), P(n)_{*}\right)
$$

is a homeomorphism of filtered $P(n)_{*}$-modules.

The Künneth formula in cohomology is a direct consequence of Theorems 4.7 and 4.2, just as in [Bo95, Thm. 4.19].

Theorem 4.8. Even for $p=2$, if $P(n)_{*}(X)$ and $P(n)_{*}(Y)$ are free $P(n)_{*}$-modules, the completed cross product

$$
\times: P(n)^{*}(X) \widehat{\otimes} P(n)^{*}(Y) \stackrel{\cong}{\longrightarrow} P(n)^{*}(X \times Y)
$$

is a homeomorphism of filtered $P(n)_{*}$-modules. 


\section{Three environments}

Three flavors of operations in $P(n)$-cohomology were encoded in [BW07] as the $P(n)_{*}$-duals of the three algebraic objects in the diagram of homomorphisms of left $P(n)_{*}$-modules (among other structures),

$$
\overline{P(n)}_{*}\left(\underline{P(n)} * \stackrel{q}{\longrightarrow} A_{*}^{*}=Q \overline{P(n)}_{*}(\underline{P(n)} * \stackrel{\sigma}{\longrightarrow} \Gamma=\overline{P(n)})_{*}(P(n)) .\right.
$$

Stable operations are dual to the Hopf algebroid $\Gamma$, which has $P(n)_{*}$-generators $a_{(i)}$ and $b_{(j)}$, described in detail in [ibid., Thm. 6.4]. Part of the structure is the right unit ring homomorphism $\eta_{R}: P(n)_{*} \rightarrow \Gamma$, which makes $\Gamma$ a right $P(n)_{*}$-module.

Additive unstable operations are dual to the bigraded Hopf algebroid $A_{*}^{*}$, where $A_{i}^{s}$ denotes the indecomposables in degree $i$ of the Hopf algebra $\left.\overline{P(n)}\right)_{*}(\underline{P(n)} s)$ and is assigned the total degree $i-s$. (It was named $Q_{*}^{*}$ in [BW07], but we wish to avoid any confusion with the homology homomorphisms $Q_{*}$ induced by the maps $Q$ in $H o$. Worse, the notation $Q \overline{P(n)}{ }_{*}(\underline{P(n)} s)$ is ambiguous; here, $Q$ denotes the indecomposables in the Hopf algebra, not the homology operation Q.) It has generators $e$, $a_{(i)}, b_{(j)}$ and $w_{k}=\eta_{R} v_{k}$, described in detail in [ibid., Thm. 7.2]. The stabilization $\sigma: A_{i}^{s} \rightarrow \Gamma_{i-s}$ has (total) degree zero and maps $a_{(i)}$ and $b_{(j)}$ to their namesakes, also $\sigma e=1$ and $\sigma w_{k}=w_{k}=\eta_{R} v_{k} \in \Gamma$.

Unstable operations are dual to the Hopf $\operatorname{ring} \overline{P(n)}_{*}(\underline{P(n)} *$ ) (not a Hopf ring in quite the ordinary sense; see $\S 6)$. It has generators $e, a_{(i)}, b_{(j)}$ and $\left[v_{k}\right]$, described in detail in [ibid., Thms. 11.1 and 11.3]; there are also useful elements $f_{i}$ (see [BW07, $(10.9)]), b_{j}$, and $[v]$ for any $v \in P(n)_{*}$. The map $q_{s}: \overline{P(n)_{*}}(\underline{P(n)} s) \rightarrow A_{*}^{s}$ simply quotients out by the $*$-decomposable elements and $P(n)_{*}$-multiples of $1_{s}$ in the Hopf algebra (with a degree shift of $-s$ ) and maps each generator to its namesake, except that $q\left[v_{k}\right]=w_{k}$.

Because the homology operation $Q$ and the homology homomorphisms $Q_{*}$ appear in so many formulae in $\S 6$, we record how they act on all three objects in diagram (24). It is also useful to include the linear functional $\langle Q,-\rangle$ that corresponds to the cohomology operation $Q$. As $q$ and $\sigma$ respect $Q, Q_{*}$ and $\langle Q,-\rangle$, we actually work mostly in the Hopf ring. A few complicated proofs have to be deferred to $\S 6$.

\section{The stable environment}

By Proposition 4.1, $\Gamma=\overline{P(n)}_{*}(P(n))$ is exactly the same set and $P(n)_{*}$-module as $P(n)_{*}(P(n))$; only the multiplication is different. The homology homomorphism $Q_{*}$ is automatically $P(n)_{*}$-linear. By Proposition 4.3(a), the homology operation $Q$ is a derivation.

Proposition 5.1. In the Hopf algebroid $\Gamma=\overline{P(n)}_{*}(P(n))$ :

(a) $Q=Q_{*}$ is the $P(n)_{*}$-linear derivation defined on the generators by $Q a_{(i)}=0$ for $0 \leqslant i<n-1, Q a_{(n-1)}=1$, and $Q b_{(j)}=0$; also $Q w_{k}=0$.

(b) The linear functional $\langle Q,-\rangle$ is given on the generators by $\left\langle Q, a_{(i)}\right\rangle=0$ for $0 \leqslant$ $i<n-1,\left\langle Q, a_{(n-1)}\right\rangle=1$, and $\left\langle Q, b_{(j)}\right\rangle=0$. 
(c) We have the relation

$$
a_{(i)}^{2}=b_{(i+1)} \quad(\text { for } 0 \leqslant i \leqslant n-1) .
$$

(d) The multiplication on $\Gamma$ is commutative and is independent of the choice of multiplication $\phi$ or $\bar{\phi}$ on $P(n)$.

It is not obvious that the ring $\Gamma$ is commutative when $p=2$.

\section{The additive environment}

Here, $Q$ remains a $P(n)_{*}$-linear derivation, but this is far less obvious for $Q_{*}$. Now that $Q: A_{i}^{s} \rightarrow A_{i-N}^{s}$ and $Q_{*}$ have different bidegrees, they no longer coincide.

Lemma 5.2. The homology homomorphism $Q_{*}: A_{i}^{s} \rightarrow A_{i}^{s+N}$ is a derivation.

The proof will follow immediately from Theorem 6.4 or Lemma 7.4, by neglecting decomposables.

Proposition 5.3. In the bigraded Hopf algebroid $A_{*}^{*}$ :

(a) $Q$ is the $P(n)_{*}$-linear derivation defined on the generators by the equations $Q e=$ $0, Q a_{(i)}=0$ for $0 \leqslant i<n-1, Q a_{(n-1)}=e, Q b_{(j)}=0$, and $Q w_{k}=0$.

(b) $Q_{*}$ is the $P(n)_{*}$-linear derivation defined on the generators by the equations $Q_{*} e=0, Q_{*} a_{(i)}=0$ for $0 \leqslant i<n-1, Q_{*} a_{(n-1)}=b_{(0)}^{2^{n-1}}, Q_{*} b_{(j)}=0$, and $Q_{*} w_{k}$ $=0$.

(c) The linear functional $\langle Q,-\rangle$ takes the following values: $\langle Q, e\rangle=0,\left\langle Q, a_{(i)}\right\rangle=0$ for $0 \leqslant i<n-1,\left\langle Q, a_{(n-1)}\right\rangle=1,\left\langle Q, b_{(j)}\right\rangle=0$, and $\left\langle Q, w_{k}\right\rangle=0$.

(d) We have the relation

$$
a_{(i)}^{2}=b_{(i+1)} \quad(\text { for } 0 \leqslant i \leqslant n-1) .
$$

(e) The multiplication on $A_{*}^{*}$ is commutative and is independent of the choice of multiplication $\phi$ or $\bar{\phi}$ on $P(n)$.

Proof of Proposition 5.1, assuming Proposition 5.3. We copy (c) from (7). For the other parts, we simply apply the stabilization $\sigma: A_{*}^{*} \rightarrow \Gamma$ to Proposition 5.3, noting that $\sigma e=\sigma b_{(0)}=1$.

It is even less obvious that the ring $A_{*}^{*}$ is commutative for $p=2$. Although Proposition 5.3 bears a strong formal resemblance to Proposition 5.1, stable proofs do not apply. Instead, we must use Hopf ring methods.

\section{The unstable environment}

The Hopf ring $\overline{P(n)_{*}}\left(\underline{P(n)}_{*}\right)$ has two multiplications, $c * d=\mu_{*}(c \overline{\times} d)$ and $c \circ$ $d=\phi_{*}(c \overline{\times} d)$, where the maps $\mu: \underline{P(n)}_{s} \times \underline{P(n)} s \rightarrow \underline{P(n)} s$ and $\phi: \underline{P(n)}{ }_{s} \times \underline{P(n)} m$ $\rightarrow \underline{P(n)}_{s+m}$ represent, in Ho, addition and multiplication in $P(n)$-cohomology. As discussed in [BW07, $\$ 10.2]$, the two Cartan formulae (10.23) and (10.36) in [BJW95] for $r(x+y)$ and $r(x y)$ continue to hold in this context.

Here, $Q$ remains a derivation, but $Q_{*}$ does not. It will be convenient to replace the Hopf ring generator $\left[v_{k}\right]$ by $\left[v_{k}\right]-1$. 
Proposition 5.4. In the Hopf ring $\overline{P(n)}_{*}\left(\underline{P(n)}_{*}\right)$ :

(a) $Q$ is a $P(n)_{*}$-linear derivation for both multiplications,

$$
Q(c * d)=Q c * d+c * Q d, \quad Q(c \circ d)=Q c \circ d+c \circ Q d,
$$

and is given on the generators by $Q e=0, Q a_{(i)}=0$ for $0 \leqslant i<n-1, Q a_{(n-1)}$ $=e, Q b_{(j)}=0$, and $Q\left(\left[v_{k}\right]-1\right)=0$; further, $Q f_{i}=0$ for $i<2^{n}$ and $Q b_{j}=0$.

(b) $Q_{*}$ is a *-homomorphism,

$$
Q_{*}(c * d)=\left(Q_{*} c\right) *\left(Q_{*} d\right),
$$

and is given on the generators by $Q_{*} e=0, Q_{*} a_{(i)}=0$ for $0 \leqslant i<n-1$, $Q_{*} a_{(n-1)}=b_{(0)}^{\circ 2^{n-1}}, Q_{*} b_{(j)}=0$, and $Q_{*}\left(\left[v_{k}\right]-1\right)=0$; further, $Q_{*} f_{i}=0$ for $0<$ $i<2^{n}, Q_{*} b_{j}=0$ for $j>0, Q_{*}[v]=1_{-s+N}$ for any $v \in P(n)_{s}$, and $Q_{*} 1_{s}=$ $1_{s+N}$.

(c) The linear functional $\langle Q,-\rangle$ is given on generators by $\langle Q, e\rangle=0,\left\langle Q, a_{(i)}\right\rangle=0$ for $0 \leqslant i<n-1,\left\langle Q, a_{(n-1)}\right\rangle=1,\left\langle Q, b_{(j)}\right\rangle=0$, and $\left\langle Q,\left[v_{k}\right]-1\right\rangle=0$.

(d) We have the relation

$$
a_{(i)}^{\circ 2}=b_{(i+1)} \quad(\text { for } 0 \leqslant i \leqslant n-1) .
$$

(e) The effect of changing the choice of multiplication on $P(n)$ from $\phi$ to $\bar{\phi}$ is to reverse both multiplications: $c * d$ is replaced by $d * c$ and $c \circ d$ by $d \circ c$.

(f) Denote by $\bar{\psi}$ the comultiplication on the Hopf ring defined using $\bar{\phi}$ instead of $\phi$ as the multiplication on $P(n)$. Then the comultiplication is also reversed: if

$$
\psi c=\sum_{\alpha} c_{\alpha}^{\prime} \otimes c_{\alpha}^{\prime \prime}
$$

then $\bar{\psi} c=\sum_{\alpha} c_{\alpha}^{\prime \prime} \otimes c_{\alpha}^{\prime}$.

Theorem 6.9 and its accompanying examples will show that the Hopf ring is definitely not commutative or cocommutative.

Conspicuous by its absence is the formula for $Q_{*}(c \circ d)$, which is complicated; it will be the subject of Theorem 6.4. The proof of (d) is also deferred to $\S 6$. Before we prove the other parts, we need to review the coalgebra structure.

\section{The unstable coalgebra structure}

We recall the coalgebra structure $(\psi, \epsilon)$ on the Hopf ring (which we warn is unrelated to the coalgebra structures on $\Gamma$ and $A_{*}^{*}$ ). We need to know how it relates to $Q, Q_{*}$ and the two multiplications.

The counit $\epsilon: \overline{P(n)}_{*}(\underline{P(n)} *) \rightarrow P(n)_{*}$ is simply induced by the maps $\omega_{s}: \underline{P(n)} s \rightarrow$ point. There are no surprises, $\epsilon(c * d)=(\epsilon c)(\epsilon d)$ and $\epsilon(c \circ d)=(\epsilon c)(\epsilon d)$. Further, $\epsilon Q c=0$ and $\epsilon Q_{*} c=\epsilon c$. For the first of these, $\epsilon Q c=\omega_{s *} Q c=Q\left(\omega_{s *} c\right)=0$ by naturality. The second follows from $\omega_{s+N} \circ Q=\omega_{s}: \underline{P(n)} s \rightarrow$ point. 
The comultiplication $\psi$ is defined in terms of the diagonal map $\Delta: \underline{P(n)} s \rightarrow$ $\underline{P(n)} s \times \underline{P(n)} s$ as

$$
\begin{aligned}
\psi: \overline{P(n)}_{*}\left(\underline{P(n)}_{s}\right) \stackrel{\Delta_{*}}{\longrightarrow} \overline{P(n)}_{*}(\underline{P(n)} s \times \underline{P(n)} s) \\
\stackrel{\cong}{\cong} \overline{P(n)}_{*}(\underline{P(n)} s) \otimes \overline{P(n)}_{*}(\underline{P(n)} s),
\end{aligned}
$$

using the Künneth isomorphism in $\overline{P(n)}$-homology. Algebraically, equation (27) is equivalent to

$$
\Delta_{*} c=\sum_{\alpha} c_{\alpha}^{\prime} \overline{\times} c_{\alpha}^{\prime \prime}
$$

To find $\psi Q c$, we apply $Q$ to get, using naturality,

$$
\Delta_{*} Q c=Q \Delta_{*} c=\sum_{\alpha} Q\left(c_{\alpha}^{\prime} \overline{\times} c_{\alpha}^{\prime \prime}\right)=\sum_{\alpha} Q c_{\alpha}^{\prime} \overline{\times} c_{\alpha}^{\prime \prime}+\sum_{\alpha} c_{\alpha}^{\prime} \overline{\times} Q c_{\alpha}^{\prime \prime} .
$$

The analogue of (28) for $Q c$ now implies that

$$
\psi Q c=\sum_{\alpha} Q c_{\alpha}^{\prime} \otimes c_{\alpha}^{\prime \prime}+\sum_{\alpha} c_{\alpha}^{\prime} \otimes Q c_{\alpha}^{\prime \prime} .
$$

For $\psi Q_{*} c$, we use the trivial equation $\Delta \circ Q=(Q \times Q) \circ \Delta$ to write

$$
\Delta_{*} Q_{*} c=(Q \times Q)_{*} \Delta_{*} c=\sum_{\alpha}(Q \times Q)_{*}\left(c_{\alpha}^{\prime} \overline{\times} c_{\alpha}^{\prime \prime}\right)=\sum_{\alpha} Q_{*} c_{\alpha}^{\prime} \overline{\times} Q_{*} c_{\alpha}^{\prime \prime},
$$

which we similarly convert to

$$
\psi Q_{*} c=\sum_{\alpha} Q_{*} c_{\alpha}^{\prime} \otimes Q_{*} c_{\alpha}^{\prime \prime} .
$$

\section{Preliminaries for Proposition 5.4}

We begin with results on $a_{(n-1)}$ and $f_{i}$.

Lemma 5.5. We have $Q a_{(n-1)}=e, Q_{*} a_{(n-1)}=b_{(0)}^{\circ 2^{n-1}}$, and $\left\langle Q, a_{(n-1)}\right\rangle=1$. Further, $Q_{*} f_{i}=0$ for $0<i<2^{n}$.

Proof. We know from [BW07, (6.15)] that $P(n)^{*}\left(\mathbb{R} P^{2 N}\right)=P(n)_{*}[t] /\left(t^{2 N+1}\right)$. The Atiyah-Hirzebruch spectral sequence in homology also collapses; $P(n)_{*}\left(\mathbb{R} P^{2 N}\right)$ is a free $P(n)_{*}$-module with basis elements $y_{i}$ for $0 \leqslant i \leqslant 2 N$, where $y_{i}$ is dual to $t^{i}$. We may view the cohomology class $t$ as a map $t: \mathbb{R} P^{2 N} \rightarrow \underline{P(n)} 1$; in $\overline{P(n)}$-homology, it induces $t_{*} y_{i}=f_{i}$, and in particular, $t_{*} y_{1}=f_{1}=e$ and $t_{*} \overline{y_{2^{n}}}=f_{2^{n}}=a_{(n-1)}$. (Indeed, this is how $a_{(i)}$ was originally defined in [Wi84], or compare [BJW95, Prop. 10.5].)

We start from the equation $Q t=t^{2^{n}}$ in cohomology. (The only alternative is $Q t=$ 0 , which soon implies that $Q$ is identically zero.) Then dually, by Proposition 4.5(c), in homology we have $Q y_{2^{n}}=y_{1}$, and by naturality, $Q a_{(n-1)}=e$. 
For any cohomology operation $r$, we have, from [BW07, (10.9)],

$$
r(t)=\left\langle r, 1_{1}\right\rangle 1+\langle r, e\rangle t+\sum_{i=2}^{2 N}\left\langle r, f_{i}\right\rangle t^{i} .
$$

If we take $r=Q$, we see that $\left\langle Q, a_{(n-1)}\right\rangle=1$. If we replace $r$ by $r \circ Q$, we get

$$
r\left(t^{2^{n}}\right)=r(Q t)=(r \circ Q)(t)=\sum_{i=0}^{2 N}\left\langle r \circ Q, f_{i}\right\rangle t^{i}=\sum_{i=0}^{2 N}\left\langle r, Q_{*} f_{i}\right\rangle t^{i},
$$

using $\left\langle r \circ Q, f_{i}\right\rangle=\left\langle Q^{*} r, f_{i}\right\rangle=\left\langle r, Q_{*} f_{i}\right\rangle$. Since $1 \circ e=0$, the Cartan formula for $r\left(t^{2^{n}}\right)$ simplifies to

$$
r\left(t^{2^{n}}\right)=\langle r, 1\rangle+\left\langle r, e^{\circ 2^{n}}\right\rangle t^{2^{n}}+\text { higher terms. }
$$

Since this holds for all $r$, comparison of the coefficients of $t^{i}$ yields $Q_{*} f_{i}=0$ for $0<i<2^{n}$ and $Q_{*} a_{(n-1)}=Q_{*} f_{2^{n}}=e^{\circ 2^{n}}=b_{(0)}^{\circ 2^{n-1}}$.

The same techniques apply to $b_{j}$ and $[v]$, with simplifications.

Lemma 5.6. We have $Q b_{j}=0$ for all $j$ and $Q_{*} b_{j}=0$ for $j>0$. In particular, for $b_{(j)}=b_{2^{j}}$ we have $Q b_{(j)}=0$ and $Q_{*} b_{(j)}=0$.

Proof. In this case, $P(n)_{*}\left(\mathbb{C} P^{\infty}\right)$ is a free $P(n)_{*}$-module on basis elements $z_{j} \in$ $P(n)_{2 j}\left(\mathbb{C} P^{\infty}\right)$ for $j \geqslant 0$, and $P(n)^{*}\left(\mathbb{C} P^{\infty}\right)=P(n)_{*}[[x]]$, with $x^{j}$ dual to $z_{j}$. The map $x: \mathbb{C} P^{\infty} \rightarrow \underline{P(n)}{ }_{2}$ induces $x_{*} z_{j}=b_{j}$. Trivially, $Q z_{j}=0$, hence $Q b_{j}=0$.

For any cohomology operation $r$, since $Q x=0$ trivially, [BW07, (10.4)] gives

$$
r(0)=r(Q x)=(r \circ Q)(x)=\sum_{j=0}^{\infty}\left\langle r \circ Q, b_{j}\right\rangle x^{j}=\sum_{j=0}^{\infty}\left\langle r, Q_{*} b_{j}\right\rangle x^{j} .
$$

We compare with $r(0)=\langle r, 1\rangle 1$, to deduce that $Q_{*} b_{j}=0$ for $j>0$.

Lemma 5.7. For any $v \in P(n)_{s}$, we have $Q_{*}[v]=1_{-s+N}$. In particular, $Q_{*} 1_{-s}=$ $Q_{*}\left[0_{s}\right]=1_{-s+N}$ and $Q_{*}\left(\left[v_{k}\right]-1\right)=0$.

Proof. Given $v \in P(n)^{-s}$ (point) $=P(n)_{s}$, we have $[v] \in \overline{P(n)}_{0}\left(\underline{P(n)}_{-s}\right)$. Trivially, $Q v=0$ in $P(n)^{-s+N}$ (point). Then by [BW07, (10.2)],

$$
r(0)=r(Q v)=(r \circ Q)(v)=\langle r \circ Q,[v]\rangle=\left\langle r, Q_{*}[v]\right\rangle
$$

for any $r$. Comparing with $r(0)=\langle r, 1\rangle$, we deduce that $Q_{*}[v]=1_{-s+N}$.

Proof of Proposition 5.4, except (d). For (a), from (21) we have

$$
\begin{aligned}
Q(c * d) & =Q \mu_{*}(c \overline{\times} d)=\mu_{*} Q(c \overline{\times} d) \\
& =\mu_{*}(Q c \overline{\times} d+c \overline{\times} Q d)=Q c * d+c * Q d,
\end{aligned}
$$

and similarly for $Q(c \circ d)$. 
In (b), (26) depends on the fact that the cohomology operation $Q$ is additive, $Q(x+y)=Q x+Q y$. For the representing map $Q: \underline{P(n)} s \rightarrow \underline{P(n)}{ }_{s+N}$, it follows that $Q \circ \mu=\mu \circ(Q \times Q)$; then

$$
Q_{*}(c * d)=Q_{*} \mu_{*}(c \overline{\times} d)=\mu_{*}(Q \times Q)_{*}(c \overline{\times} d)=\mu_{*}\left(Q_{*} c \overline{\times} Q_{*} d\right)=Q_{*} c * Q_{*} d .
$$

Lemmas 5.5, 5.6 and 5.7 take care of many of the statements. For $Q e$, we may write $Q e=Q Q a_{(n-1)}=0$ (which is trivial except when $n=1$ ). The remaining values are trivial, as they lie in groups that are zero.

Part (e) was proved in [BW07, §10.1].

For (f), we use $T \circ \Delta=\Delta: \underline{P(n)}_{k} \rightarrow \underline{P(n)}_{k} \times \underline{P(n)}_{k}$. Equation (28) yields

$$
\Delta_{*} c=T_{*} \Delta_{*} c=\sum_{\alpha} T_{*}\left(c_{\alpha}^{\prime} \overline{\times} c_{\alpha}^{\prime \prime}\right)=\sum_{\alpha} c_{\alpha}^{\prime \prime} \times c_{\alpha}^{\prime},
$$

with the help of (19). The analogue of (28) for $\bar{\psi}$ translates this into $\bar{\psi} c=\sum_{\alpha} c_{\alpha}^{\prime \prime} \otimes c_{\alpha}^{\prime}$, as required.

Proof of Proposition 5.3 except (e), assuming Lemma 5.2. We apply the quotient $\operatorname{map} q: \overline{P(n)} *(\underline{P(n)} *) \rightarrow A_{*}^{*}$ to Proposition 5.4 and use $q\left(\left[v_{k}\right]-1\right)=w_{k}$.

The two missing items will be proved in $\S 6$. The proofs of Propositions 5.3(e) and 5.4(d) will be applications following Proposition 7.2.

\section{Structure of the Hopf Ring}

Here, we explain in detail what kind of object the Hopf ring $\overline{P(n)}_{*}\left(\underline{P(n)}_{*}\right)$ is when $p=2$. Because the multiplication on $P(n)$ is noncommutative, several of the Hopf ring axioms require modification. Whenever two spaces are shuffled, equation (19) introduces extra terms. We exhibit only the four affected axioms and the three rules for (co)commutation. All the other axioms listed in [BJW95, §10] survive unaltered. Several of the more complicated proofs are deferred to the next section.

From now on, as we are almost exclusively concerned with the Hopf ring, we simplify the notation. We write $H(s)$ for the Hopf algebra $\overline{P(n)_{*}}\left(\frac{P(n)}{s}\right), H(s)_{i}$ for the group of elements of degree $i$ in $H(s)$, and $H(*)$ for the whole Hopf ring $\overline{P(n)}_{*}(\underline{P(n)} *)$.

In this section, we need only limited information from [BW07, §11] on the Hopf ring. As noted earlier, we replace each generator $\left[v_{k}\right]$ by $\left[v_{k}\right]-1$, to make all our o-generators lie in the augmentation ideal $\operatorname{Ker} \epsilon$.

(i) We start with the o-generators $e, a_{(i)}, b_{(j)}$ and $\left[v_{k}\right]-1$;

(ii) A o-monomial is any o-product of o-generators (including the empty product $\left.[1]-1_{0} \in H(0)\right)$;

(iii) A *o-monomial is any $*$-product of o-monomials (including the empty product $1_{s} \in H(s)$ for each $\left.s\right)$.

To stay inside $\operatorname{Ker} \epsilon$, we follow the convention of [RW77], that for any element 
$d \in \operatorname{Ker} \epsilon$, we define $d^{\circ 0}=[1]-1_{0}$, with the result that $d^{\circ 0} \circ d=d$ still holds. Corollary 6.8 will verify that the $* \circ-$ monomials do indeed span the Hopf ring $H(*)$ as a $P(n)_{*}$-module.

\section{The coalgebra structure}

Theorem 6.1. For any elements $c, d \in H(s)=\overline{P(n)}_{*}\left(\underline{P(n)}_{s}\right)$, where $\psi c$ is given by equation (27) and similarly $\psi d=\sum_{\beta} d_{\beta}^{\prime} \otimes d_{\beta}^{\prime \prime}$, we have

$$
\psi(c * d)=\sum_{\alpha} \sum_{\beta} c_{\alpha}^{\prime} * d_{\beta}^{\prime} \otimes c_{\alpha}^{\prime \prime} * d_{\beta}^{\prime \prime}+\sum_{\alpha} \sum_{\beta} v_{n} c_{\alpha}^{\prime} * Q d_{\beta}^{\prime} \otimes Q c_{\alpha}^{\prime \prime} * d_{\beta}^{\prime \prime} .
$$

Remark 6.2. We could arrange the formula to read simply $\psi(c * d)=(\psi c) *(\psi d)$, which states that $\psi$ preserves $*$-products, by endowing the tensor product $H(s) \otimes$ $H(s)$ with the nonstandard $*$-multiplication suggested by [BW07, (2.6)],

$$
\left(c \otimes c^{\prime}\right) *\left(d \otimes d^{\prime}\right)=c * d \otimes c^{\prime} * d^{\prime}+v_{n} c * Q d \otimes Q c^{\prime} * d^{\prime} .
$$

It will be useful to note that we do not need formulae involving the elements $1_{j}$, because for any $c \in H(s)$ we have, as usual,

$$
1_{s} * c=c=c * 1_{s}, \quad 1_{m} \circ c=(\epsilon c) 1_{s+m}=c \circ 1_{m}, \quad \psi 1_{s}=1_{s} \otimes 1_{s}, \quad \epsilon 1_{s}=1 .
$$

We therefore concentrate attention on elements of the augmentation ideal $\operatorname{Ker} \epsilon$. Because $\epsilon$ is the counit, we may rewrite $\psi c$ for $c \in \operatorname{Ker} \epsilon$ in the more useful form

$$
\psi c=c \otimes 1+\sum_{i} c_{i}^{\prime} \otimes c_{i}^{\prime \prime}+1 \otimes c,
$$

breaking out the two end terms, where $\epsilon c_{i}^{\prime}=0$ and $\epsilon c_{i}^{\prime \prime}=0$ for all $i$, and similarly

$$
\psi d=d \otimes 1+\sum_{j} d_{j}^{\prime} \otimes d_{j}^{\prime \prime}+1 \otimes d .
$$

Then equation (28) is replaced by

$$
\Delta_{*} c=c \overline{\times} 1+\sum_{i} c_{i}^{\prime} \overline{\times} c_{i}^{\prime \prime}+1 \overline{\times} c .
$$

Theorem 6.3. Given elements $c$ and $d$ of the Hopf ring that satisfy $\epsilon c=0$ and $\epsilon d=$ 0 , with $\psi c$ and $\psi d$ given by (33) and and (34), we have

$$
\begin{aligned}
\psi(c \circ d)= & c \circ d \otimes 1+\sum_{i} \sum_{j} c_{i}^{\prime} \circ d_{j}^{\prime} \otimes c_{i}^{\prime \prime} \circ d_{j}^{\prime \prime}+1 \otimes c \circ d \\
& +\sum_{i} \sum_{j} v_{n} c_{i}^{\prime} \circ Q d_{j}^{\prime} \otimes Q c_{i}^{\prime \prime} \circ d_{j}^{\prime \prime} .
\end{aligned}
$$

The previous remark applies equally well here.

Now we can state the formula for $Q_{*}(c \circ d)$, deferred from $\S 5$.

Theorem 6.4. Given elements $c$ and $d$ of the Hopf ring that satisfy $\epsilon c=0$ and $\epsilon d=$ 0 , with $\psi c$ and $\psi d$ given by (33) and (34), the action of $Q_{*}$ on o-products is given by

$$
Q_{*}(c \circ d)=Q_{*} c \circ d+\sum_{i} \sum_{j}\left(c_{i}^{\prime} \circ Q_{*} d_{j}^{\prime}\right) *\left(Q_{*} c_{i}^{\prime \prime} \circ d_{j}^{\prime \prime}\right)+c \circ Q_{*} d .
$$




\section{The distributive laws}

Because the distributive laws involve some shuffling of factors, they have to be modified.

Theorem 6.5. For any elements $a, b, c$ in $\overline{P(n)}_{*}\left(\underline{P(n)}_{*}\right)$ that satisfy $\epsilon a=0, \epsilon b=0$ and $\epsilon c=0$, with $\psi c$ given by (33), the distributive laws are

$$
(a * b) \circ c=\sum_{i}\left(a \circ c_{i}^{\prime}\right) *\left(b \circ c_{i}^{\prime \prime}\right)+\sum_{i} v_{n}\left(a \circ Q c_{i}^{\prime}\right) *\left(Q b \circ c_{i}^{\prime \prime}\right)
$$

and

$$
c \circ(a * b)=\sum_{i}\left(c_{i}^{\prime} \circ a\right) *\left(c_{i}^{\prime \prime} \circ b\right)+\sum_{i} v_{n}\left(c_{i}^{\prime} \circ Q a\right) *\left(Q c_{i}^{\prime \prime} \circ b\right) .
$$

It is clear that the Hopf ring element $a_{(n-1)}$ causes most of our difficulties.

Definition 6.6. Let us call a o-monomial or other expression in our o-generators wild if it explicitly contains the element $a_{(n-1)}$, or tame if it does not.

Proposition 6.7. Let $c$ and $d$ be *o-monomials as in in (31)(iii).

(a) $c \circ d$ is a $P(n)_{*}$-linear combination of $* \circ-$ monomials. If $c$ and $d$ are tame, so is $c \circ d$.

(b) If $c \neq 1, \psi c$ has the form (33), where every $c_{i}^{\prime}$ and $c_{i}^{\prime \prime}$ is a $P(n)_{*}$-linear combination of $* 0-m o n o m i a l s$. If $c$ is tame, so are every $c_{i}^{\prime}$ and $c_{i}^{\prime \prime}$.

(c) Qc is a sum of *o-monomials, and is zero if $c$ is tame.

(d) $Q_{*} c$ is a $P(n)_{*}$-linear combination of $* 0$-monomials, and is zero if $c$ is tame and $c \neq 1$.

By (a), the set of linear combinations of *o-monomials is closed under o-multiplication as well as $*$-multiplication. We know from [BW07] that the o-generators generate $H(*)$ as a Hopf ring over $P(n)_{*}$.

Corollary 6.8. The *o-monomials (31)(iii) span the Hopf ring $H(*)$ as a $P(n)_{*-}$ module.

Proof of Proposition 6.7. We assume the previous four theorems (of which none is yet proved). None of the formulae we use introduces an $a_{(n-1)}$ where there was not one before, so tameness is preserved. Part (c) follows directly from Proposition 5.4(a) by induction.

We prove the other three parts together, by induction on degree. In degree zero, we have only the o-generators $\left[v_{k}\right]-1$, which are easily handled by the standard formulae $[v] *\left[v^{\prime}\right]=\left[v+v^{\prime}\right]$ and $[v] \circ\left[v^{\prime}\right]=\left[v v^{\prime}\right]$ for $v, v^{\prime} \in P(n)_{*}$, and the formula 
for $\psi([v]-1)$, below. For $(\mathrm{b})$, we start from the formulae in [BW07],

$$
\begin{aligned}
\psi e & =e \otimes 1+1 \otimes e, \\
\psi a_{(i)} & =\sum_{j+k=2^{i+1}} f_{j} \otimes f_{k}, \\
\psi b_{(i)} & =\sum_{j+k=2^{i}} b_{j} \otimes b_{k}, \\
\psi([v]-1)= & ([v]-1) \otimes 1+([v]-1) \otimes([v]-1) \\
& \quad+1 \otimes([v]-1) \quad\left(\text { for any } v \in P(n)_{*}\right),
\end{aligned}
$$

where $f_{j}$ is a $*$-product of selected generators $a_{(k)}$, also (if $j$ is odd) $e$, and $b_{j}$ is a (complicated) linear combination of $*$ o-monomials in the generators $b_{(k)}$ and $\left[v_{k}\right]-1$. Then we use Theorem 6.3 for $\psi(c \circ d)$ and Theorem 6.1 for $\psi(c * d)$. For (a), we use the distributive laws in Theorem 6.5 to expand $(z * c) \circ d$ and $c \circ(z * d)$, where $z$ is a o-monomial. For (d), we use Theorem 6.4 and Proposition 5.4(b).

\section{The commutation rules}

Neither multiplication is commutative in this Hopf ring environment, nor is the comultiplication cocommutative.

Theorem 6.9. Given elements of the Hopf ring $c, d \in \overline{P(n)}{ }_{*}(\underline{P(n)} *)$ that satisfy $\epsilon c=0$ and $\epsilon d=0$, with $\psi c$ and $\psi d$ given by (33) and (34), we have the following (co) commutation rules:

(a) For the comultiplication $\psi$,

$$
\sum_{i} c_{i}^{\prime} \otimes c_{i}^{\prime \prime}=\sum_{i} c_{i}^{\prime \prime} \otimes c_{i}^{\prime}+\sum_{i} v_{n} Q c_{i}^{\prime \prime} \otimes Q c_{i}^{\prime}
$$

(b) For the *-multiplication,

$$
d * c=c * d+v_{n} Q c * Q d
$$

(c) For the o-multiplication,

$$
\begin{aligned}
d \circ c= & c \circ d+v_{n} Q c \circ Q d+Q_{*} c \circ Q_{*} d \circ\left[v_{n}\right] \\
& +\sum_{i} \sum_{j}\left(c_{i}^{\prime} \circ d_{j}^{\prime}\right) *\left(Q_{*} c_{i}^{\prime \prime} \circ Q_{*} d_{j}^{\prime \prime} \circ\left[v_{n}\right]\right) \\
& +\sum_{i} \sum_{j} v_{n}\left(Q c_{i}^{\prime} \circ Q d_{j}^{\prime}\right) *\left(Q_{*} c_{i}^{\prime \prime} \circ Q_{*} d_{j}^{\prime \prime} \circ\left[v_{n}\right]\right) .
\end{aligned}
$$

Nevertheless, we shall see in Proposition 7.2 that all tame elements in the Hopf ring are o-central.

Remark 6.10. Of course $a_{(n-1)}$ commutes with itself, but equation (42) yields

$$
a_{(n-1)} \circ a_{(n-1)}=a_{(n-1)} \circ a_{(n-1)}+v_{n} e \circ e+b_{(0)}^{\circ 2^{n-1}} \circ b_{(0)}^{\circ 2^{n-1}} \circ\left[v_{n}\right] .
$$

This is consistent, because by $[\mathbf{B W 0 7}, \S 10.5]$, the main relation $\left(\mathcal{R}_{n}\right)$ is $v_{n} b_{(0)}=$ $b_{(0)}^{\circ 2^{n}} \circ\left[v_{n}\right]$ and $e \circ e=b_{(0)}$. 
Remark 6.11. We present concrete examples of non(co)commutativity.

It is easy to see that $*$ is not commutative in general. If we take $c=a_{(n-1)} \circ b_{(0)}$ and $d=a_{(n-1)} \circ b_{(1)}$, equation (41) shows directly that

$$
d * c=c * d+v_{n}\left(e \circ b_{(0)}\right) *\left(e \circ b_{(1)}\right) .
$$

It is less obvious that o-multiplication is not commutative. If we set $c=(e \circ$ $\left.a_{(n-1)}\right) * b_{(0)}$ and $d=\left(e \circ a_{(n-1)}\right) * b_{(1)}$, equation (42) reduces to

$$
d \circ c=c \circ d+v_{n}\left(b_{(0)} \circ b_{(1)}\right) * b_{(0)}^{\circ 2} .
$$

Alternatively, we may reduce $c \circ d$ and $d \circ c$ to standard form as in [BW07, 111.3 ]. Since $e \circ a_{(n-1)}$ and $b_{(0)}=e \circ e$ are both suspensions and therefore primitive, equation (32) yields

$$
\psi c=c \otimes 1+e \circ a_{(n-1)} \otimes b_{(0)}+b_{(0)} \otimes e \circ a_{(n-1)}+1 \otimes c .
$$

Then the distributive law (38) yields, after some simplification,

$$
d \circ c=\left(b_{(0)} \circ b_{(n)}\right) *\left(b_{(0)} \circ b_{(1)}\right)+\left(e \circ a_{(n-1)} \circ b_{(0)}\right) *\left(e \circ a_{(n-1)} \circ b_{(1)}\right),
$$

with no extra term, while for $c \circ d,(39)$ yields the same, plus the extra term $v_{n} b_{(0)}^{\circ 2} *$ $\left(b_{(0)} \circ b_{(1)}\right)$.

Neither is the comultiplication cocommutative. If we take (as above) $c=(e \circ$ $\left.a_{(n-1)}\right) * b_{(0)}$ and $d=\left(e \circ a_{(n-1)}\right) * b_{(1)}$ and use equation (32) to compute $\psi(c * d)$ and $\psi(d * c)$, we find that both contain all the same terms one would normally expect, which are symmetric. In addition, $\psi(c * d)$ has two extra terms $v_{n} b_{(0)}^{* 3} \otimes$ $b_{(0)}^{* 2}+v_{n} b_{(0)}^{* 2} \otimes b_{(0)} * b_{(1)}$ that are not symmetric, whereas $\psi(d * c)$ has $v_{n} b_{(0)} * b_{(1)} \otimes$ $b_{(0)}^{* 2}+v_{n} b_{(0)}^{* 2} \otimes b_{(0)}^{* 3}$.

\section{Proofs of the Hopf ring structure}

We establish the remaining theorems in $\S 6$. All our proofs follow the same pattern (already seen in $\S 5$ ): we represent some relation in $P(n)$-cohomology in the category Ho, apply $\overline{P(n)}$-homology, and evaluate on a generic twisted cross product.

A diagonal map $\Delta$ introduces the comultiplication by way of equation (35). Whenever two factors are switched, equation (19) adds extra terms involving $Q$. (Some of these extra terms will in due course go away, but we do not know which in advance.)

Proof of Theorems 6.1 and 6.3. To abbreviate, we write $W=\underline{P(n)}$ s. The maps

$$
W \times W \stackrel{\mu}{\longrightarrow} W \stackrel{\Delta}{\longrightarrow} W \times W
$$

and

$$
\begin{aligned}
& W \times W \stackrel{\Delta \times \Delta}{\longrightarrow} W \times W \times W \times W \\
& \stackrel{\mathrm{id} \times T \times \mathrm{id}}{\longrightarrow} W \times W \times W \times W \stackrel{\mu \times \mu}{\longrightarrow} W \times W
\end{aligned}
$$

trivially coincide. We apply $\overline{P(n)}$-homology and evaluate on the element $c \overline{\times} d \in$ $\overline{P(n)}_{*}(\underline{P(n)} s \times \underline{P(n)} s)$, using (35) and (19) to obtain (32).

The proof of (36) is completely analogous, with $\phi$ replacing $\mu$. 
Proof of Theorem 6.5. We represent the distributive law $(x+y) z=x z+y z$ in $P(n)$ cohomology by the equality of the maps

$$
\underline{P(n)}_{s} \times \underline{P(n)}_{s} \times \underline{P(n)}_{m} \stackrel{\mu \times \mathrm{id}}{\longrightarrow} \underline{P(n)} s \times \underline{P(n)}_{m} \stackrel{\phi}{\longrightarrow} \underline{P(n)} s+m
$$

and

$$
\begin{aligned}
& \underline{P(n)}_{s} \times \underline{P(n)}_{s} \times \underline{P(n)}_{m} \stackrel{\mathrm{id} \times \mathrm{id} \times \Delta}{\longrightarrow} \underline{P(n)} s \times \underline{P(n)}_{s} \times \underline{P(n)}_{m} \times \underline{P(n)} m \\
& \stackrel{\mathrm{id} \times T \times \mathrm{id}}{\longrightarrow} \underline{P(n)} \underline{s} \times \underline{P(n)}_{m} \times \underline{P(n)}_{s} \times \underline{P(n)}_{m} \\
& \stackrel{\phi \times \phi}{\longrightarrow} \underline{P(n)}_{s+m} \times \underline{P(n)}_{s+m} \stackrel{\mu}{\longrightarrow} \underline{P(n)} s+m
\end{aligned}
$$

We apply $\overline{P(n)}$-homology to $a \overline{\times} b \overline{\times} c \in \overline{P(n)})_{*}\left(\underline{P(n)}_{s} \times \underline{P(n)}_{s} \times \underline{P(n)}_{m}\right)$ to obtain the distributive law (38). The other distributive law is analogous.

We next establish a commutation rule for $d \circ c$ that will in due course reduce to equation (42).

Lemma 7.1. Assuming that $\epsilon c=0$ and $\epsilon d=0$, with $\psi c$ and $\psi d$ given by equations (33) and (34), we have

$$
\begin{aligned}
d \circ c= & c \circ d+v_{n} Q c \circ Q d+Q_{*} c \circ Q_{*} d \circ\left[v_{n}\right]+v_{n} Q_{*} Q c \circ Q_{*} Q d \circ\left[v_{n}\right] \\
& +\sum_{i, j}\left(c_{i}^{\prime} \circ d_{j}^{\prime}\right) *\left(Q_{*} c_{i}^{\prime \prime} \circ Q_{*} d_{j}^{\prime \prime} \circ\left[v_{n}\right]\right) \\
& +\sum_{i, j} v_{n}\left(Q c_{i}^{\prime} \circ Q d_{j}^{\prime}\right) *\left(Q_{*} c_{i}^{\prime \prime} \circ Q_{*} d_{j}^{\prime \prime} \circ\left[v_{n}\right]\right) \\
& +\sum_{i, j} v_{n}\left(c_{i}^{\prime} \circ d_{j}^{\prime}\right) *\left(Q_{*} Q c_{i}^{\prime \prime} \circ Q_{*} Q d_{j}^{\prime \prime} \circ\left[v_{n}\right]\right) \\
& +\sum_{i, j} v_{n}^{2}\left(Q c_{i}^{\prime} \circ Q d_{j}^{\prime}\right) *\left(Q_{*} Q c_{i}^{\prime \prime} \circ Q_{*} Q d_{j}^{\prime \prime} \circ\left[v_{n}\right]\right) \\
& +\sum_{i, j} v_{n}\left(Q c_{i}^{\prime} \circ d_{j}^{\prime}\right) *\left(Q_{*} c_{i}^{\prime \prime} \circ Q_{*} Q d_{j}^{\prime \prime} \circ\left[v_{n}\right]\right) .
\end{aligned}
$$

Proof. We rewrite $(20)$ as $y x=x y+(Q x)(Q y) v_{n}$, and represent it in Ho by the maps $\phi: \underline{P(n)}_{m} \times \underline{P(n)}_{s} \rightarrow \underline{P(n)}_{s+m}$ and

$$
\begin{aligned}
& \underline{P(n)}_{m} \times \underline{P(n)}_{s} \stackrel{\Delta \times \Delta}{\longrightarrow} \underline{P(n)}_{m} \times \underline{P(n)}_{m} \times \underline{P(n)}_{s} \times \underline{P(n)}_{s} \\
& \stackrel{\mathrm{id} \times T \times \mathrm{id}}{\longrightarrow} \underline{P(n)} \underline{m}_{m} \times \underline{P(n)}_{s} \times \underline{P(n)}_{m} \times \underline{P(n)} s \\
& \stackrel{T \times T}{\longrightarrow} \underline{P(n)} s \times \underline{P(n)}_{m} \times \underline{P(n)} s \times \underline{P(n)} m \\
& =\underline{P(n)}_{s} \times \underline{P(n)}_{m} \times \underline{P(n)}_{s} \times \underline{P(n)}_{m} \times \text { point } \\
& \stackrel{\mathrm{id} \times \mathrm{id} \times Q \times Q \times v_{n}}{\longrightarrow} \\
& \underline{P(n)}_{s} \times \underline{P(n)}_{m} \times \underline{P(n)}_{s+N} \times \underline{P(n)}_{m+N} \times \underline{P(n)}_{-2 N} \\
& \stackrel{\phi \times \phi_{3}}{\longrightarrow} \underline{P(n)} \underline{s+m} \times \underline{P(n)}_{s+m} \stackrel{\mu}{\longrightarrow} \underline{P(n)} s+m .
\end{aligned}
$$


We apply $\overline{P(n)}$-homology and evaluate on $d \overline{\times} c \in \overline{P(n)_{*}}\left(\underline{P(n)}_{m} \times \underline{P(n)} s\right)$, using $Q \circ Q=0, Q_{*} 1=1, \epsilon Q c=0$ and $\epsilon Q_{*} c=\epsilon c$. The result is (43).

Proposition 7.2. Every tame element of the Hopf ring $H(*)$ is o-central.

Proof. We take $d$ in (43) to be any of our o-generators other than $a_{(n-1)}$. By (40) and Proposition 5.4, $Q d, Q_{*} d$ and each $Q_{*} d_{j}^{\prime \prime}$ all vanish, so that (43) simplifies to $d \circ c=c \circ d$.

If $c_{1}$ and $c_{2}$ are both tame and o-central, so is $c_{1} \circ c_{2}$, trivially. To see that $c_{1} * c_{2}$ is also o-central, we compare the two distributive laws (39) and (38) (which have now been proved) for any $d \in \operatorname{Ker} \epsilon$, with $\psi d$ expressed as in (34),

$$
d \circ\left(c_{1} * c_{2}\right)=\sum_{j}\left(d_{j}^{\prime} \circ c_{1}\right) *\left(d_{j}^{\prime \prime} \circ c_{2}\right)=\sum_{j}\left(c_{1} \circ d_{j}^{\prime}\right) *\left(c_{2} \circ d_{j}^{\prime \prime}\right)=\left(c_{1} * c_{2}\right) \circ d .
$$

It follows that any tame expression is o-central.

Proof of Proposition 5.3(e). All our o-generators (31)(i) commute with each other, as $a_{(n-1)}$ is the only wild one. When we pass to the indecomposables $A_{*}^{*}$ of the Hopf ring, the o-generators map to $e, a_{(i)}, b_{(j)}$ and $w_{k}$, which therefore still commute and generate the $P(n)_{*}$-algebra $A_{*}^{*}$.

From Proposition 5.4(e), switching the multiplication on $P(n)$ from $\phi$ to $\bar{\phi}$ reverses the o-multiplication, which has no effect on $A_{*}^{*}$.

Remark 7.3. As another example of noncommutativity, we note that Proposition 7.2 does not extend to the elements $f_{i}$ for $i>2^{n}$, which are wild. Take $c=f_{2^{n}+1}=$ $e * a_{(n-1)}$ and $d=f_{2^{n}+2}=a_{(0)} * a_{(n-1)}$, where $n \geqslant 2$. (If $n=1, a_{(0)} * a_{(n-1)}=0$ and $f_{4}$ does not exist.) We compute as before that

$$
f_{2^{n}+2} \circ f_{2^{n}+1}=f_{2^{n}+1} \circ f_{2^{n}+2}+v_{n} b_{(0)} *\left(e \circ a_{(0)}\right) .
$$

Proof of Proposition 5.4(d). This is similar to the stable proof of (25) in [BW07, (6.17)], and in fact relies on it.

We apply the Cartan formula to (29) to obtain

$$
r\left(t^{2}\right)=\sum_{i=0}^{2 N} \sum_{j=0}^{2 N}\left\langle r, f_{i} \circ f_{j}\right\rangle t^{i+j}
$$

for all operations $r$. Now $t^{2}$ is the Chern class of the complexified real Hopf line bundle

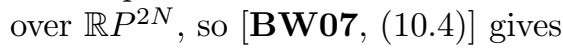

$$
r\left(t^{2}\right)=\sum_{k=0}^{N}\left\langle r, b_{k}\right\rangle t^{2 k} .
$$

Comparing coefficients of $t^{2 k}$, we deduce that $b_{k}=\sum_{i+j=2 k} f_{i} \circ f_{j}$. As the elements $f_{i}$ for $i<2^{n}$ are tame and so o-commute by Proposition 7.2, this simplifies to $b_{k}=f_{k}^{\circ 2}$, provided $2 k \leqslant 2^{n}$; in particular, $a_{(i)}^{\circ 2}=b_{(i+1)}$ for $0 \leqslant i \leqslant n-2$.

This proof is not available for $a_{(n-1)}^{\circ 2}$ in $\overline{P(n)_{*}}\left(\underline{P(n)}_{*}\right)$, as $t^{2^{n+1}}=0$, but it does show that $a_{(n-1)}^{\circ 2}=b_{(n)}$ in $\overline{P(n+1)}_{*}(\underline{P(n+1)} 2)$. The homomorphism of Hopf rings 
$H(*) \rightarrow \overline{P(n+1)}_{*}\left(\underline{P(n+1)}_{*}\right)$ induced by the canonical map $\rho: P(n) \rightarrow P(n+1)$ carries each generator of $H(*)$ to its namesake in $\overline{P(n+1)}_{*}(\underline{P(n+1)} *)$. It also adds one new generator, $a_{(n)}$, and kills the ideal $\left(v_{n}\right)$ in $P(n)_{*}$. The only nonzero element of $H(2)_{2^{n+1}}$ that lies in Ker $\rho_{*}$ is $v_{n} b_{(0)}$. We must have $a_{(n-1)}^{\circ 2}=b_{(n)}$ in $H(2)$, as the only other candidate, $a_{(n-1)}^{\circ 2}=b_{(n)}+v_{n} b_{(0)}$, does not stabilize correctly to (25).

We next prove a similarly extended version of (37).

Lemma 7.4. Given $c, d \in H(*)$ that satisfy $\epsilon c=0$ and $\epsilon d=0$, with $\psi c$ and $\psi d$ given by (33) and (34), we have

$$
\begin{aligned}
Q_{*}(c \circ d)= & Q_{*} c \circ d+c \circ Q_{*} d \\
& +\sum_{i, j}\left(c_{i}^{\prime} \circ Q_{*} d_{j}^{\prime}\right) *\left(Q_{*} c_{i}^{\prime \prime} \circ d_{j}^{\prime \prime}\right) \\
& +\sum_{i, j} v_{n}\left(c_{i}^{\prime} \circ Q_{*} Q d_{j}^{\prime}\right) *\left(Q_{*} Q c_{i}^{\prime \prime} \circ d_{j}^{\prime \prime}\right)
\end{aligned}
$$

Proof. We represent the derivation formula $Q(x y)=x(Q y)+(Q x) y$ in $P(n)$-cohomology unstably by equality of the two maps

$$
\underline{P(n)}_{s} \times \underline{P(n)}_{m} \stackrel{\phi}{\longrightarrow} \underline{P(n)}_{s+m} \stackrel{Q}{\longrightarrow} \underline{P(n)}_{s+m+N}
$$

and

$$
\begin{aligned}
& \underline{P(n)}_{s} \times \underline{P(n)}_{m} \stackrel{\Delta \times \Delta}{\longrightarrow} \underline{P(n)}_{s} \times \underline{P(n)}_{s} \times \underline{P(n)}_{m} \times \underline{P(n)}_{m} \\
& \stackrel{\mathrm{id} \times T \times \mathrm{id}}{\longrightarrow} \underline{P(n)} \underline{\mathrm{in}}_{s} \times \underline{P(n)}_{m} \times \underline{P(n)}_{s} \times \underline{P(n)}_{m} \\
& \stackrel{\text { id } \times Q \times Q \times \text { id }}{\longrightarrow} \underline{P(n)}_{s} \times \underline{P(n)}_{m+N} \times \underline{P(n)}_{s+N} \times \underline{P(n)}_{m} \\
& \stackrel{\phi \times \phi}{\longrightarrow} \underline{P(n)}{ }_{s+m+N} \times \underline{P(n)}_{s+m+N} \stackrel{\mu}{\longrightarrow} \underline{P(n)}{ }_{s+m+N} .
\end{aligned}
$$

Again, we apply $\overline{P(n)}$-homology and evaluate on $c \overline{\times} d \in \overline{P(n)}_{*}\left(\underline{P(n)}_{s} \times \underline{P(n)}_{m}\right)$, to obtain (44).

Lemma 7.5. Take any element $c$ in the Hopf ring.

(a) If $g$ is any of our o-generators (31)(i) other than $a_{(n-1)}$, we have $Q_{*}(c \circ g)=$ $\left(Q_{*} c\right) \circ g$.

(b) For $g=a_{(n-1)}$, we have

$$
Q_{*}\left(c \circ a_{(n-1)}\right)=\left(Q_{*} c\right) \circ a_{(n-1)}+c \circ b_{(0)}^{\circ 2^{n-1}} .
$$

Proof. We may assume $c \in \operatorname{Ker} \epsilon$, as everything is zero if $c=1$. We put $d=g$ in (44). Since all the $Q_{*} d_{j}^{\prime}$ and $Q d_{j}^{\prime}$ vanish by (40) and Proposition 5.4, also $Q_{*} d$ in case (a), equation (44) simplifies as stated.

Corollary 7.6. Let $z$ be $a \circ$-monomial as in (31)(ii). Then $Q_{*} z$ is tame if $z$ is wild, and $Q_{*} z=0$ if $z$ is tame. 
Proof. If $z$ is tame, $Q_{*} z=0$ from Lemma 7.5(a), by induction on the number of o-factors in $z$. If $z$ is wild, now that we know from Proposition 7.2 that all our ogenerators o-commute $\left(a_{(n-1)}\right.$ is the only wild generator), we may shuffle all factors $a_{(n-1)}$ to the end. We use the relation (now proved) $a_{(n-1)}^{\circ 2}=b_{(n)}$ to reduce the number of factors $a_{(n-1)}$ until we have at most one. If now $z=z^{\prime} \circ a_{(n-1)}$, with $z^{\prime}$ tame, Lemma 7.5(b) shows that $Q_{*} z=z^{\prime} \circ b_{(0)}^{\circ 2^{n-1}}$, which is tame.

Lemma 7.7. For all $*$ o-monomials $c$ we have $Q_{*} Q c=Q Q_{*} c=0$.

Proof. By naturality of the homology operation $Q$, we have $Q_{*} Q c=Q Q_{*} c$. We show that $Q Q_{*} c=0$.

The result is trivial for $c=1$. Otherwise, we may write $c=z_{1} * z_{2} * \cdots * z_{r}$, where each $z_{i}$ is a o-monomial and $r>0$. By Corollary 7.6, each $Q_{*} z_{i}$ is tame (if not zero). Then Proposition 5.4 shows that (i) each $Q Q_{*} z_{i}=0$, (ii) $Q_{*} c=Q_{*} z_{1} * Q_{*} z_{2} * \cdots *$ $Q_{*} z_{r}$, and hence (iii) $Q Q_{*} c=0$.

Proof of Theorem 6.4. Lemma 7.7 allows us to omit the unwanted terms in (44) to obtain (37).

Proof of Theorem 6.9. For (a), we deduce from (30) that

$$
\Delta_{*} c=1 \times c+\sum_{i} c_{i}^{\prime \prime} \times c_{i}^{\prime}+c \times 1,
$$

after replacing (27) by (33). By (18), we can rewrite $c_{i}^{\prime \prime} \times c_{i}^{\prime}$ as $c_{i}^{\prime \prime} \overline{\times} c_{i}^{\prime}+v_{n} Q c_{i}^{\prime \prime} \overline{\times} Q c_{i}^{\prime}$. (For the end terms we have simply $1 \times c=1 \overline{\times} c$ and $c \times 1=c \overline{\times}$ 1.) We compare with (35).

For (b), we represent the relation $y+x=x+y$ in $P(n)$-cohomology by the equality of maps $\mu=\mu \circ T: \underline{P(n)} s \times \underline{P(n)} s \rightarrow \underline{P(n)} s$. We apply $\overline{P(n)}$-homology to these

maps and evaluate on $d \overline{\times} c \in \overline{P(n)}_{*}(\underline{P(n)} s \times \underline{P(n)} s)$ to obtain (41), with the help of (19).

For (c), Lemma 7.7 allows us to omit all the unwanted terms in Lemma 7.1 to obtain (42).

\section{A nonadditive splitting}

To complete the proof of Lemma 5.1 in [BW07], given $m \geqslant n$, we need a nonadditive idempotent operation $\theta(m)$ on the ungraded cohomology theory $P(n)^{M}(-)$, where $M=g(n, m)$ is the numerical function given by

$$
g(n, m)=2\left(2^{n}+2^{n+1}+\cdots+2^{m}\right) .
$$

(We know there is no relevant additive idempotent in this dimension.) We shall use $\theta(m)$ to produce what we actually want, a natural splitting $\overline{\theta(m)}: P(n, m)^{M}(-) \rightarrow$ $P(n)^{M}(-)$ of the canonical projection $\rho(m): P(n)^{*}(-) \rightarrow P(n, m)^{*}(-)$; we recall from [BW07] that $P(n, m)$ is the spectrum constructed from $P(n)$ to have the homotopy groups $P(n, m)_{*}=P(n)_{*} / J_{m}$, where $J_{m}$ denotes the ideal $\left(v_{m+1}, v_{m+2}, \ldots\right) \subset$ $P(n)_{*}$. 
We shall define $\theta(m)$ by its linear functional $\langle\theta(m),-\rangle: H(M) \rightarrow P(n)_{*}$ on the Hopf algebra $H(M)=\overline{P(n)}_{*}\left(\underline{P(n)}_{M}\right)$. In this section, we give axioms for $\langle\theta(m),-\rangle$ that ensure that $\theta(m)$ has the desired properties; in the following section, Corollary 9.4 actually constructs the linear functional.

\section{A Hopf ring ideal}

Definition 8.1. Denote by $\mathfrak{J}_{m}$ the $*$-ideal in the Hopf ring $H(*)$ generated by all elements of the form $c \circ\left(\left[v_{k}\right]-1\right)$ with $c \in H(*)$ and $k>m$.

As in [BW07, Lemma 19.35], this is a Hopf ring ideal. It is closely related to the ideal $J_{m}$. Following [BJW95, (23.6)], the axioms we need on $\langle\theta(m),-\rangle$ are:

(i) $\langle\theta(m), c\rangle=0$ for all $c \in \mathfrak{J}_{m} \cap H(M)$;

(ii) $\langle\theta(m), c\rangle \equiv \epsilon_{A} q_{M} c \bmod J_{m}$ for all $c \in H(M)$;

where we recall the projection $q_{M}: \overline{P(n)}_{i}\left(\underline{P(n)}_{M}\right) \rightarrow A_{i}^{M}$ and the additive counit $\epsilon_{A}: A_{i}^{M} \rightarrow P(n)_{i-M}$. These axioms imply that $\theta(m)$ has the desired properties.

First, $\theta(m)$ behaves correctly on the homotopy groups $\pi_{*}\left(\underline{P(n)}_{M}\right)=\Sigma^{M} P(n)_{*}$.

Lemma 8.2. If $\theta(m)$ satisfies the axioms (46), then on the homotopy groups we have

(i) $\theta(m)_{*} \Sigma^{M} v=0$ for all $v \in J_{m}$;

(ii) $\theta(m)_{*} \Sigma^{M} v \equiv \Sigma^{M} v \bmod J_{m}$ for all $v \in P(n)_{*}$.

Proof. By [BW07, (12.1)], given $v \in P(n)_{s}$,

$$
\theta(m)_{*} \Sigma^{M} v=\Sigma^{M}\left\langle\theta(m), e^{\circ M+s} \circ([v]-1)\right\rangle,
$$

where we make use of $e \circ 1=0$. As $v \in J_{m}$ implies that $[v]-1$ lies in $\mathfrak{J}_{m}$, we can read off the results from (46).

Next, we study the homology homomorphism $\theta(m)_{*}: H(M) \rightarrow H(M)$ induced by $\theta(m): \underline{P(n)}_{M} \rightarrow \underline{P(n)}_{M}$.

Lemma 8.3. If $\theta(m)$ satisfies the axioms (46), then on the $\overline{P(n)}$-homology groups $H(M)=\overline{P(n)_{*}}\left(\underline{P(n)}_{M}\right)$ we have

(i) $\theta(m)_{*} c=0$ for all $c \in \mathfrak{J}_{m} \cap H(M)$;

(ii) $\theta(m)_{*} c \equiv c \bmod \mathfrak{J}_{m}$ for all $c \in H(M)$.

Proof. The proof of the similar result Lemma 23.4 in [BJW95] applies, with one minor modification. An additional case is needed to handle the extra generators $a_{(i)}$. Proposition 10.3(vii) of [BW07] allows us to treat these generators the same way as the others.

It is now easy to deduce that $\theta(m)_{*}$ and hence $\theta(m)$ are idempotent. However, in the nonadditive context, this is not enough; a more sophisticated relation is needed, from Lemma 3.10 in [Bo95].

Lemma 8.4. If $\theta=\theta(m)$ satisfies the axioms (46), it satisfies the identity

$$
\theta(x+y-\theta(y))=\theta(x) \quad\left(\text { in } P(n)^{M}(-)\right) .
$$


Proof. We repeat a technique from $\S 6$, but in reverse: we use a Hopf algebra calculation to establish the relation (47) in cohomology. The right side, considered as a function of $(x, y)$, is represented by the map

$$
W \times W \stackrel{\mathrm{id} \times \omega_{M}}{\longrightarrow} W \times \text { point }=W \stackrel{\theta}{\longrightarrow} W,
$$

where we abbreviate by writing $W=\underline{P(n)}{ }_{M}$. We apply $\overline{P(n)}$-homology and evaluate on $c \overline{\times} d$ to get the result $\theta_{*}((\epsilon d) c)$.

The left side is represented by the composite

$$
\begin{gathered}
W \times W \stackrel{\mathrm{id} \times \Delta}{\longrightarrow} W \times W \times W \stackrel{\operatorname{id} \times \operatorname{id} \times \theta}{\longrightarrow} W \times W \times W \\
\stackrel{\operatorname{id} \times \operatorname{id} \times \nu}{\longrightarrow} W \times W \times W \stackrel{\mu_{3}}{\longrightarrow} W \stackrel{\theta}{\longrightarrow} W
\end{gathered}
$$

where $\nu$ represents the operation "-" and induces the conjugation $\nu_{*}=\chi$ on $H(M)$. Again, we apply $\overline{P(n)}$-homology and evaluate on $c \overline{\times} d$. If we omit the final $\theta$, we find

$$
\sum_{\beta} c * d_{\beta}^{\prime} * \chi \theta_{*} d_{\beta}^{\prime \prime} \equiv c * \sum_{\beta} d_{\beta}^{\prime} * \chi d_{\beta}^{\prime \prime}=c *(\epsilon d) 1=(\epsilon d) c \bmod \mathfrak{J}_{m}
$$

using Lemma 8.3(ii). When we apply $\theta_{*}$, which kills $\mathfrak{J}_{m} \cap H(M)$ by Lemma 8.3(i), we get $\theta_{*}((\epsilon d) c)$.

The other needed relation, $\theta(0)=0$, is automatic here, as $P(n)^{M}$ (point) $=0$. As in [Bo95], the two relations have several immediate consequences:

(i) $\theta(\theta(y))=\theta(y)$;

(ii) $\theta(x+y)=\theta(x)$ if $\theta(y)=0$;

(iii) $\theta(y-\theta(y))=0$;

(iv) $\operatorname{Ker} \theta=\{x: \theta(x)=0\}$ is a subgroup of $P(n)^{M}(-)$.

For (i), we put $x=\theta(y)$ in (47), to show that $\theta$ is idempotent in the ordinary sense. If $\theta(y)=0,(47)$ simplifies to (ii). For (iii) we put $x=0$. For (iv), we have $0 \in \operatorname{Ker} \theta$ by assumption. If $x$ and $y$ lie in $\operatorname{Ker} \theta$, so does $x+y$ by (ii). Given $\theta(y)=0$, we put $x=-y$ in (47) to see that $\theta(-y)=0$, to finish (iv).

By (iv), we can legitimately form the quotient group $\operatorname{Coim} \theta=P(n)^{M}(-) / \operatorname{Ker} \theta$ (not to be confused with the image of $\theta$, which is not a subgroup of $P(n)^{M}(-)$ ); its elements are $\operatorname{cosets} x+\operatorname{Ker} \theta$. It is easy to show as in [Bo95, §3], using equations (48), that $\operatorname{Ker} \theta$ and $\operatorname{Coim} \theta$ are ungraded cohomology theories (in the sense of [Bo95, Lemma 3.10$]$ ), representable in $H o$ by $H$-spaces. We build the commutative diagram of ungraded cohomology theories and natural transformations

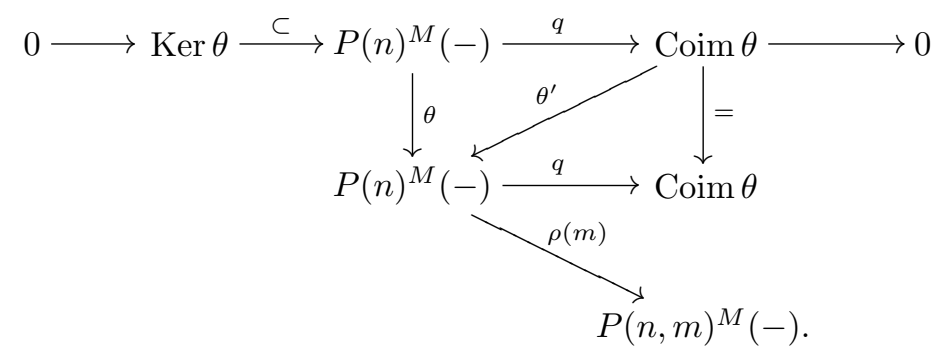


It features a short exact sequence. The non-additive operation $\theta^{\prime}$ is defined by $\theta^{\prime}(x+$ $\operatorname{Ker} \theta)=\theta(x)$ to make the upper triangle commute; by (48)(ii), it is well defined. The lower triangle commutes because $\theta(x)+\operatorname{Ker} \theta=x+\operatorname{Ker} \theta$ by (iii).

We really want a splitting of $\rho(m)$, not $q$. By Lemma 8.2, the natural transformation $\rho(m) \circ \theta^{\prime}$ induces an isomorphism on spheres and hence generally. (Equivalently, the map that represents $\rho(m) \circ \theta^{\prime}$ induces an isomorphism of homotopy groups and is therefore a homotopy equivalence.) We define

$$
\overline{\theta(m)}=\theta^{\prime} \circ\left(\rho(m) \circ \theta^{\prime}\right)^{-1}: P(n, m)^{M}(-) \longrightarrow P(n)^{M}(-) .
$$

Trivially, we have $\rho(m) \circ \overline{\theta(m)}=\mathrm{id}$ and $\overline{\theta(m)} \circ \rho(m)$ is an idempotent operation on $P(n)^{M}(-)$. (Lemma 8.2 strongly suggests that $\rho(m) \circ \theta=\rho(m)$, which readily implies that $\overline{\theta(m)} \circ \rho(m)=\theta$; however, we do not need this, and a complete proof appears to require properties of the spectrum $P(n, m)$ beyond the scope of this paper.)

\section{Reduction to standard form}

Before we produce the linear functional $\langle\theta(m),-\rangle$ for $\S 8$ in Corollary 9.4, we need much more information on the Hopf ring $H(*)$. Specifically, we need a precise description of how the ideal $\mathfrak{J}_{m}$ sits inside $H(*)$. Our strategy is quite different and significantly simpler than the proof of the analogous result for $B P$ given in [BJW95, §23], and more closely resembles the proof of the additive case [BW07, Lemma 9.3].

We first need to refine Corollary 6.8. As all our o-generators o-commute by Proposition 7.2 and we have the relations $e^{\circ 2}=b_{(0)}$ and $a_{(i)}^{\circ 2}=b_{(i+1)}$, any o-monomial may be written

$$
\begin{aligned}
z= & e^{\circ h} \circ a^{\circ I} \circ b^{\circ J} \circ\left(\left[v^{K}\right]-1\right) \\
= & e^{\circ h} \circ a_{(0)}^{\circ i_{0}} \circ a_{(1)}^{\circ i_{1}} \circ \cdots \circ a_{(n-1)}^{\circ i_{n-1}} \circ b_{(0)}^{\circ j_{0}} \circ b_{(1)}^{\circ j_{1}} \circ b_{(2)}^{\circ j_{2}} \circ \ldots \\
& \circ\left(\left[v_{n}\right]-1\right)^{\circ k_{n}} \circ\left(\left[v_{n+1}\right]-1\right)^{\circ k_{n+1}} \circ\left(\left[v_{n+2}\right]-1\right)^{\circ k_{n+2}} \circ \ldots,
\end{aligned}
$$

as in [BW07, (11.1)], with multi-indices $I=\left(i_{0}, i_{1}, \ldots, i_{n-1}\right), J=\left(j_{0}, j_{1}, j_{2}, \ldots\right)$ and $K=\left(k_{n}, k_{n+1}, k_{n+2}, \ldots\right)$, where $h$ and every $i_{r}$ is 0 or 1 . (We used the identity $([v]-1) \circ\left(\left[v^{\prime}\right]-1\right)=\left[v v^{\prime}\right]-1$ to make the notation less cumbersome. Of course, we could also replace $\left[v^{K}\right]-1$ by simply $\left[v^{K}\right]$, as long as $e$ or some $a_{(i)}$ or $b_{(j)}$ is present, but we prefer to stay within the augmentation ideal $\operatorname{Ker} \epsilon$ as much as possible.) We need to know how $Q$ acts; by Proposition 5.4,

$$
\begin{aligned}
& Q\left(e^{\circ h} \circ a^{\circ I, 0} \circ b^{\circ J} \circ\left(\left[v^{K}\right]-1\right)\right)=0, \\
& Q\left(e^{\circ h} \circ a^{\circ I, 1} \circ b^{\circ J} \circ\left(\left[v^{K}\right]-1\right)\right)=e \circ e^{\circ h} \circ a^{\circ I, 0} \circ b^{\circ J} \circ\left(\left[v^{K}\right]-1\right),
\end{aligned}
$$

where $I=\left(i_{0}, i_{1}, \ldots, i_{n-2}\right)$ and $a^{\circ I, s}$ denotes $a_{(0)}^{\circ i_{0}} \circ a_{(1)}^{\circ i_{1}} \circ \cdots \circ a_{(n-2)}^{\circ i_{n-2}} \circ a_{(n-1)}^{\circ s}$.

We note that each of our o-generators lies in a group $H(s)_{i}$ for which $2 i-s>0$; it follows that each $H(s)_{i}$ contains only finitely many o-monomials, and hence only finitely many $*$ o-monomials.

In [BW07, Defn. 8.1], we defined certain o-monomials as being allowable; the others are not (in the end) needed. (The empty o-monomial $e^{\circ 0} \circ a^{\circ 0} \circ b^{\circ 0} \circ([1]-$ $1)=[1]-1_{0} \in H(0)$ is allowable.) Given $s$, we choose a total ordering of all the 
allowable o-monomials that lie in $H(s)$; then we call the $*$ o-monomial (in $H(s)$ )

$$
c=z_{1} * z_{2} * \cdots * z_{r}
$$

an ordered $*$ o-monomial if each $z_{i}$ is allowable and $z_{1}<z_{2}<\cdots<z_{r}$. This allows us to rephrase Theorem 11.3 of [BW07] in the following form.

Theorem 9.1. For each $s$, the Hopf algebra $H(s)=\overline{P(n)_{*}}(\underline{P(n)} s)$ is a free $P(n)_{*}$ module, with a basis consisting of all the ordered $*$ o-monomials (51) that lie in $H(s)$ (including the empty $*$-product $1_{s}$ ).

The rest of the section will be concerned with reducing a general $*$ o-monomial to this standard form. (This is not as straightforward as [BW07] suggests.) Our proofs will make it clear that the choice of ordering is not significant.

We show that the ideal $\mathfrak{J}_{m}$ reduces to what one would like, but only in a certain range. Let us call the o-monomial (49) a $\mathfrak{J}_{m}$-monomial if it visibly lies in $\mathfrak{J}_{m}$, i.e. $v^{K} \in J_{m}$ (has a factor $v_{k}$ with $k>m$ ).

Theorem 9.2. If $s \leqslant M=g(n, m)$, the $*$-ideal $\mathfrak{J}_{m} \cap H(s)$ is the free $P(n)_{*}$-submodule of $H(s)$ with a basis consisting of all the ordered $* 0$-monomials (51) in which at least one $*$-factor $z_{i}$ is a $\mathfrak{J}_{m}$-monomial.

Remark 9.3. What distinguishes this from [BW07, Lemma 9.3] when $p=2$ is that if $s=M$, some allowable $\mathfrak{J}_{m}$-monomials fail to be $Q$-allowable and so do not appear there, namely those of the form

$$
b_{(0)}^{\circ N} \circ b_{(0)}^{\circ 2^{n+1}} \circ b_{\left(d_{n+1}\right)}^{\circ 2^{n+2}} \circ d_{\left(d_{n+2}\right)}^{\circ 2^{n+3}} \circ \cdots \circ b_{\left(d_{m}\right)}^{\circ 2^{m+1}} \circ\left[v_{m+1}\right],
$$

where $0 \leqslant d_{n+1} \leqslant d_{n+2} \leqslant \cdots \leqslant d_{m}$.

Corollary 9.4. There exists a $P(n)_{*}$-linear functional $\langle\theta(m),-\rangle: H(M) \rightarrow P(n)_{*}$ that satisfies the axioms (46).

Proof. We simply choose $\langle\theta(m), c\rangle \in P(n)_{*}$ for each ordered $*$ o-monomial $c$ in the form (51). If some $z_{i}$ is a $\mathfrak{J}_{m}$-monomial, we must take $\langle\theta(m), c\rangle=0$ to satisfy (46)(i); then (ii) is automatic, because $\epsilon_{A} q_{M} c$ lies in $J_{m}$ if $r=1$, or is zero if $r>1$. Otherwise, we simply choose $\langle\theta(m), c\rangle$ to satisfy axiom (ii).

\section{Reduction of o-monomials}

We show how to reduce a general o-monomial (49) to allowable o-monomials. Our starting point is [BW07, Thm. 8.2], except that we temporarily exclude the o-generators $a_{(i)}$. We adapt it to the Hopf ring environment by including the decomposables.

Lemma 9.5. Any $\circ-$ monomial $z=e^{\circ h} \circ b^{\circ J} \circ\left(\left[v^{K}\right]-1\right)$ can be expressed as a $P(n)_{*}$ linear combination of:

(i) Allowable o-monomials $z^{\prime}$;

(ii) Decomposable $*$-products of two or more o-monomials.

None of the resulting expressions contains an $a_{(i)}$. 
Proof. This result is actually inherited from $B P$. It uses only the main relations $\left(\mathcal{R}_{k}\right)$ and $\left(\mathcal{R}_{n}^{\prime}\right)$ in [BW07], so does not introduce any $a_{(i)}$. We do not need to include 1 in (ii), as $z \in \operatorname{Ker} \epsilon$.

In (i), we could restrict $z^{\prime}$ to be $Q$-allowable, but this would not be compatible with Theorem 9.1. We next reinstate the $a^{\circ I}$. We note that the definition of allowable (unlike $Q$-allowable) makes no reference to the factor $a^{\circ I}$. We recall from Definition 6.6 that a Hopf ring expression in our o-generators is wild if it contains $a_{(n-1)}$ or tame if it does not. For tame elements, Proposition 6.7 simplifies as follows.

Lemma 9.6. Suppose $c$ and $d$ are tame $* \circ$-monomials, where $c \neq 1$. Then:

(a) $Q_{*} c=0$;

(b) $\operatorname{cod}$ is a $P(n)_{*}$-linear combination of tame *o-monomials;

(c) $\psi c=c \otimes 1+\sum_{i} c_{i}^{\prime} \otimes c_{i}^{\prime \prime}+1 \otimes c$, where every $c_{i}^{\prime}$ and $c_{i}^{\prime \prime}$ is a $P(n)_{*}$-linear combination of tame $*$ o-monomials.

Lemma 9.7. Any o-monomial $z=e^{\circ h} \circ a^{\circ I} \circ b^{\circ J} \circ\left(\left[v^{K}\right]-1\right)$ can be expressed as $a$ $P(n)_{*}$-linear combination of:

(i) Allowable o-monomials $z^{\prime}$, which are wild if and only if $z$ is wild;

(ii) *-products of two or more tame o-monomials.

We can recursively apply the lemma to the monomials appearing in (ii).

Corollary 9.8. Any tame o-monomial can be expressed as a $P(n)_{*}$-linear combination of $*$-products of one or more tame allowable o-monomials.

Proof of Lemma 9.7. We apply Lemma 9.5 to $e^{\circ h} \circ b^{\circ J} \circ\left(\left[v^{K}\right]-1\right)$ and then o-multiply by $a^{\circ I}$. For (i), this is obvious, as the extra o-factor $a^{\circ I}$ does not affect allowability. For (ii), we put back the o-factors $a_{(i)}$ one at a time, using the distributive law

$$
a_{(i)} \circ\left(c_{1} * c_{2}\right)=\sum_{j=1}^{2^{i+1}-1}\left(f_{j} \circ c_{1}\right) *\left(f_{2^{i+1}-j} \circ c_{2}\right) .
$$

We observe that $f_{2^{n}}=a_{(n-1)}$ never appears, even if $i=n-1$. We apply Lemma 9.6 to both $*$-factors on the right.

\section{Reduction of $\mathfrak{J}_{m}$-monomials}

We particularly need to know what happens when we reduce a $\mathfrak{J}_{m}$-monomial.

As in [BW07], we define the b-length of the o-monomial (49) as $\sum_{r} j_{r}$, the total number of o-factors of the form $b_{(j)}$ (including repetitions). We note that in Definition 8.1 of [BW07], the disallowed monomials of type (i) have $b$-length at least $\frac{1}{2} g(n, q)$, while those of type (ii) have $b$-length at least $\frac{1}{2} g(n, q)-1$, with $g(n, q)$ given by equation (45). Because we are dealing with allowable rather than $Q$-allowable monomials, the other three types are irrelevant. This prepares us for the main lemma.

Lemma 9.9. Provided $s \leqslant g(n, m)$, any $\mathfrak{J}_{m}$-monomial $z \in H(s)$ can be expressed as a $P(n)_{*}$-linear combination of:

(i) Allowable $\mathfrak{J}_{m}$-monomials $z^{\prime}$, which are wild if and only if $z$ is wild; 
(ii) *-products of two or more tame o-monomials, of which at least one is a $\mathfrak{J}_{m}$-monomial.

Just as we did for Corollary 9.8, we can recursively apply Corollary 9.8 and Lemma 9.9 to the o-monomials appearing in (ii).

Corollary 9.10. If $s \leqslant g(n, m)$, any tame $\mathfrak{J}_{m}$-monomial in $H(s)$ can be expressed as a $P(n)_{*}$-linear combination of:

(i) Tame allowable $\mathfrak{J}_{m}$-monomials;

(ii) *-products of two or more tame allowable o-monomials, of which at least one is a $\mathfrak{J}_{m}$-monomial.

Proof of Lemma 9.9. We do not apply Lemma 9.7 directly. Any $\mathfrak{J}_{m}$-monomial has the form $z=y \circ\left(\left[v_{q}\right]-1\right)$ for some $q>m$ and we apply that lemma to $y$ instead, replacing $y$ by an allowable o-monomial $y^{\prime}$ or a $*$-product $c=z_{1} * z_{2} * \cdots * z_{r}$ of tame o-monomials $z_{i}$.

In the second case, we write $c=d * z_{r}$, where $d=z_{1} * z_{2} * \cdots * z_{r-1}$. Then by equations (38) and (40),

$$
\begin{aligned}
c \circ\left(\left[v_{q}\right]-1\right)= & \left(d \circ\left(\left[v_{q}\right]-1\right)\right) * z_{r}+\left(d \circ\left(\left[v_{q}\right]-1\right)\right) *\left(z_{r} \circ\left(\left[v_{q}\right]-1\right)\right) \\
& +d *\left(z_{r} \circ\left(\left[v_{q}\right]-1\right)\right),
\end{aligned}
$$

which shows by induction on $r$ that $c \circ\left(\left[v_{q}\right]-1\right)$ has the required form.

In the first case, we fix $s$ and an arbitrarily large degree ceiling $i_{0}$, and show by downward induction on $h$ that the lemma holds for all $z^{\prime}=y^{\prime} \circ\left(\left[v_{h}\right]-1\right)$ with $y^{\prime}$ allowable that lie in $H(s)$ and have degree at most $i_{0}$. This statement is true vacuously for large enough $h$ (depending on $s$ and $i_{0}$ ), as there are only finitely many o-monomials in each $H(s)_{i}$. We assume it holds for all $h>q$ and prove it holds for $h=q$, assuming that $q>m$.

Case 1: $y^{\prime}$ has no factor $e$ or $\left[v_{k}\right]-1$.

Since $y^{\prime} \in H\left(s+2\left(2^{q}-1\right)\right)$, the $b$-length of $z^{\prime}$ is at most

$$
\frac{1}{2}\left(s+2\left(2^{q}-1\right)\right) \leqslant \frac{1}{2} g(n, m)+2^{q}-1 \leqslant \frac{1}{2} g(n, q)-1,
$$

allowing for possible factors $a_{(i)}$. Thus $z^{\prime}$ is not a disallowed monomial of type (i); nor is it of type (ii), as it has no factor $e$. Therefore $z^{\prime}$ is allowable.

Case 2: $y^{\prime}$ has a factor e, but no factor $\left[v_{k}\right]-1$.

This time, the $b$-length of $z^{\prime}$ is at most

$$
\frac{1}{2}\left(s-1+2\left(2^{q}-1\right)\right)<\frac{1}{2} g(n, q)-1
$$

and is therefore allowable.

Case 3: $y^{\prime}=u \circ\left(\left[v_{k}\right]-1\right)$, where $k \leqslant q$.

Since $y^{\prime}$ is allowable, $z^{\prime}=u \circ\left(\left[v_{k}\right]-1\right) \circ\left(\left[v_{q}\right]-1\right)$ remains allowable, by the form of [BW07, Defn. 8.1]. 
Case 4: $y^{\prime}=u \circ\left(\left[v_{k}\right]-1\right)$, where $k>q$.

Then $z^{\prime}=\left(u \circ\left(\left[v_{q}\right]-1\right)\right) \circ\left(\left[v_{k}\right]-1\right)$, to which we apply the induction hypothesis.

Lemma 9.11. If $s \leqslant g(n, m)$, the *-ideal $\mathfrak{J}_{m} \cap H(s)$ in $H(s)$ is generated by the allowable $\mathfrak{J}_{m}$-monomials that lie in $H(s)$.

Proof. Take a typical generator $c \circ\left(\left[v_{q}\right]-1\right)$ of $\mathfrak{J}_{m}$. Since $1 \circ\left(\left[v_{q}\right]-1\right)=0$, we may assume $c \in \operatorname{Ker} \epsilon$. By Theorem 9.1, it is enough to consider the case $c=z_{1} * z_{2} *$ $\cdots * z_{r}$, with $r>0$ and each $z_{i}$ a o-monomial. By (52) and induction on $r$, the ideal $\mathfrak{J}_{m} \cap H(s)$ is generated by elements of the form $z \circ\left(\left[v_{q}\right]-1\right)$ with $z$ a o-monomial and $q>m$. We apply Lemma 9.9 and Corollary 9.10 to $z \circ\left(\left[v_{q}\right]-1\right)$.

\section{Reduction of $* 0$-monomials}

We assume from now on that $s \leqslant g(n, m)$. We take any $*$-product $c$ of wild allowable o-monomials and tame o-monomials (whether allowable or not) that lie in $H(s)$; by Theorem 9.1 and Lemma 9.7, these generate $H(s)$ as a $P(n)_{*}$-module. Since any tame element is *-central by Proposition $6.7(\mathrm{c})$ and Theorem $6.9(\mathrm{~b})$, we may write

$$
c=z_{1} * z_{2} * \cdots * z_{r} * T,
$$

where each $z_{i}$ is a wild allowable o-monomial and $T$ is a $P(n)_{*}$-linear combination of tame *o-monomials.

Our object is to reduce such $c$ to the basis in Theorem 9.1. There are three steps: we must shuffle the factors $z_{i}$ into their chosen order, deal with repeated wild factors, and finally expand $T$ (which does not affect anything else).

Lemma 9.12. Given $c$ as in equation (53), suppose $z_{i}>z_{i+1}$. Then

$$
\begin{aligned}
c= & z_{1} * \ldots z_{i-1} * z_{i+1} * z_{i} * z_{i+2} * \cdots * z_{r} * T \\
& +z_{1} * \cdots * z_{i-1} * z_{i+2} * \cdots * z_{r} * T^{\prime},
\end{aligned}
$$

where $T^{\prime}=v_{n} Q z_{i+1} * Q z_{i} * T$ is tame.

If $z_{i}$ (or $\left.z_{i+1}\right)$ is a $\mathfrak{\mathfrak { J }}_{m}$-monomial, so is $Q z_{i}\left(\right.$ or $\left.Q z_{i+1}\right)$.

Proof. We plug in (41), $z_{i} * z_{i+1}=z_{i+1} * z_{i}+v_{n} Q z_{i+1} * Q z_{i}$. By (50), $Q z_{i}$ and $Q z_{i+1}$ are always tame o-monomials (though the extra $e$ could disallow them). Moreover, if $z$ is a $\mathfrak{J}_{m}$-monomial, so is $Q z$.

Lemma 9.13. Given $c$ as in equation (53), suppose that $z_{i}=z_{i+1}=e^{\circ h} \circ a^{\circ I, 1} \circ$ $b^{\circ J} \circ\left(\left[v^{K}\right]-1\right)$ as in $(49)$. Then:

(a) If $i_{n-2}=0, c=z_{1} * \cdots * z_{i-1} * z_{i+2} \cdots * z_{r} * T^{\prime}$, where $T^{\prime}$ has the form $\left(z^{\prime}+\right.$ $\left.v_{n} z^{\prime \prime}\right) * T$ and is tame. If $z_{i}$ is a $\mathfrak{J}_{m}$-monomial, so are $z^{\prime}$ and $z^{\prime \prime}$.

(b) If $i_{n-2}=1$, c is a $P(n)_{*}$-linear combination of terms of the forms

$$
\begin{aligned}
& z_{1} * \cdots * z_{i-1} * z_{i}^{\prime} * z_{i+2} * \cdots * z_{r} * T, \\
& z_{1} * \cdots * z_{i-1} * z_{i+2} * \cdots * z_{r} * T^{\prime}, \\
& z_{1} * \cdots * z_{i-1} * z^{\prime \prime} * z_{i+2} * \cdots * z_{r} * T,
\end{aligned}
$$

where $z_{i}^{\prime}$ and $z^{\prime \prime}$ are wild allowable and $T^{\prime}=t * T$ is tame. If $z_{i}$ is a $\mathfrak{J}_{m^{-}}$ monomial, so are $z_{i}^{\prime}, z^{\prime \prime}$ and $t$. 
Proof. We plug in the formula (11.6) or (11.7) from [BW07] for $F z_{i}=z_{i} * z_{i}$, which (in either case) has the form $F z_{i}=z^{\prime}+v_{n} z^{\prime \prime}$, where $z^{\prime \prime}$ happens to be allowable. If $z_{i}$ is a $\mathfrak{J}_{m}$-monomial, so are $z^{\prime}$ and $z^{\prime \prime}$.

If $i_{n-2}=0, z^{\prime}$ and $z^{\prime \prime}$ are both tame, and we have (a).

If $i_{n-2}=1, z^{\prime}$ and $z^{\prime \prime}$ are both wild. By Lemma $9.7, z^{\prime}$ is a linear combination of wild allowable o-monomials $z_{i}^{\prime}$ and a linear combination $t$ of tame $*$ o-monomials. By Lemma 9.9 , if $z^{\prime}$ is a $\mathfrak{J}_{m}$-monomial, so are $z_{i}^{\prime}$ and each term of $t$. This completes (b).

We apply Lemmas 9.12 and 9.13 to (53) as often as possible; the process must terminate after a finite number of steps, as each application reduces the count $r$ of wild $*$-factors $z_{i}$ or improves the ordering of the wild $*$-factors (without changing $r$ ). The result is a linear combination of terms of the form (53) in which $z_{1} * z_{2} * \cdots * z_{r}$ is an ordered $*$ o-monomial.

The final step is to expand the tame factor $T$ by Corollary 9.8 as a linear combination of $*$-products $T^{\prime}=t_{1} * t_{2} * \cdots * t_{k}$ of tame allowable o-monomials. As each $t_{i}$ is $*$-central, we can shuffle the factors $t_{i}$ in the product $z_{1} * z_{2} * \cdots * z_{r} * T^{\prime}$ anywhere, to place all the $*$-factors in non-decreasing order, without introducing any extra terms. If any $t_{i}$ is repeated in a term, $[\mathbf{B W 0 7}, \S 11.4]$ shows that $F t_{i}=t_{i} * t_{i}=0$, which kills that term.

Proof of Theorem 9.2. By Lemma 9.11 and Theorem 9.1, every element of $\mathfrak{J}_{m} \cap$ $H(s)$ is a linear combination of $*$-products (53) in which at least one $*$-factor is a $\mathfrak{J}_{m}$-monomial. This property is preserved by each application of Lemma 9.12 or Lemma 9.13; thus each $c$ is transformed into a linear combination of ordered *omonomials $c^{\prime}$ having the same property, as required.

\section{References}

[Ad69] J. F. Adams, Lectures on generalised cohomology, Lecture Notes in Mathematics 99, Springer-Verlag, Berlin, 1969, 1-138.

[Bo95] J. M. Boardman, Chapter 14: Stable operations in generalized cohomology, in Handbook of Algebraic Topology (ed. I.M. James), Elsevier, Amsterdam, 1995, 585-686.

[BJW95] J. M. Boardman, D. C. Johnson, W. S. Wilson, Chapter 15: Unstable operations in generalized cohomology, in Handbook of Algebraic Topology (ed. I.M. James), Elsevier, Amsterdam, 1995, 687-828.

[BW07] J. M. Boardman, W. S. Wilson, $k(n)$-torsion-free $H$-spaces and $P(n)$ cohomology, Canad. J. Math. 59 (2007), 1154-1206.

[Mi78] O. K. Mironov, Multiplications in cobordism theories with singularities, and Steenrod-tom Dieck operations, Math. USSR-Izv. 13 (1979), 89106, tr. from Izv. Akad. Nauk SSSR Ser. Mat. 42 (1978).

[Na02] C. Nassau, On the structure of $P(n)_{*}(P(n))$ for $p=2$, Trans. Amer. Math. Soc. 354 (2002), 1749-1757.

[RW77] D. C. Ravenel, W. S. Wilson, The Hopf ring for complex cobordism, J. Pure Appl. Algebra 9 (1977), 241-280. 
[US69] United States Geological Survey (1969), Virginia: Guinea and Woodford quadrangles.

[Wi84] W. S. Wilson, The Hopf ring for Morava K-theory, Publ. Res. Inst. Math. Sci., Kyoto Univ. 20 (1984), 1025-1036.

J. Michael Boardman boardman@math.jhu.edu

Department of Mathematics, Johns Hopkins University, 3400 N. Charles St., Baltimore MD 21218-2686, U.S.A. 